\title{
Competencia financiera y educación: una aproximación desde el mapeo de la ciencia
}

\section{Financial competence and education: a science mapping approach}

\section{F. Javier Sánchez Antolín (javisanchez@colegiolourdes.es)}

Colegio Nuestra Señora de Lourdes - Valladolid (España)

José M. Marbán (josemaria.marban@uva.es)

https://orcid.org/0000-0002-6561-6784

\author{
Facultad de Educación y Trabajo Social, Universidad de Valladolid (España) \\ http://dx.doi.org/10.12795/EDUCADE.2021.i12.02
}

\begin{abstract}
RESUMEN: El constructo Competencia Financiera ha recibido atención por parte de la comunidad científica desde mediados del siglo pasado, pero las publicaciones en las que el mismo se enmarca en un contexto educativo son mucho más recientes, contexto que fija ahora su atención en un nuevo constructo, el de Alfabetización Financiera. El presente estudio ofrece una visión general de la evolución y del estado actual de la investigación vinculada a ambos por medio de un mapeo sistemático y con la mirada puesta en el ámbito propio de la educación, mapeo apoyado en el software de análisis bibliométrico VOSviewer. Los resultados muestran cómo las publicaciones enmarcadas en el ámbito educativo se han empezado a mostrar relevantes principalmente en el último quinquenio y cómo las temáticas más relevantes, aunque muestran una dispersión alta y en continuo crecimiento, hacen referencia a la educación financiera, a las cualificaciones y titulaciones profesionales, al análisis de riesgos en diversos escenarios, a la evaluación o a la toma de decisiones. Por último, llama la atención la escasa relación que muestran los trabajos centrados en la Competencia Financiera con aquellos orientados a la Educación Financiera, especialmente si tenemos en cuenta el elevado número de estudios y trabajos desarrollados en los últimos años en este segundo ámbito.
\end{abstract}

PALABRAS CLAVE: Alfabetización Financiera, Competencia Financiera, Educación Financiera, Mapeo de la Ciencia.

\begin{abstract}
Financial Competence construct has received attention from the scientific community since the middle of the last century, but publications in which it is framed in an educational context are much more recent, a context that now focuses its attention on a new construct, named Financial Literacy. This study offers an overview of the evolution and current state of research linked to both constructs by means of a systematic mapping and with an eye on the field of education itself, mapping supported by the bibliometric analysis software VOSviewer. The results show how publications framed in the educational field have begun to be considered relevant mainly in the last five years and how the most relevant topics, although they show a high dispersion and in continuous growth, refer to financial education, qualifications and professional qualifications, risk analysis in various scenarios, evaluation, or decision-making. Finally, the scarce relationship that the studies focused on Financial Competence show with those oriented to Financial Education is striking, especially if we take into account the high number of studies and works developed in recent years in this second area.
\end{abstract}

KEYWORDS: Financial Competence, Financial Education, Financial Literacy, Science mapping

Artículo de investigación. Recibido: 05-04-21 - Aceptado: 22-06-21

Licencia Creative Commons BY NC ND · $2021 \cdot$ Universidad de Sevilla - AECA 


\section{INTRODUCCIÓN}

La educación en competencias (Delors \& UNESCO, 1996: cap. 3) está siendo en los últimos años un tema recurrente en los cambios educativos, tanto desde un punto de vista metodológico como de legislación educativa. Entre las competencias, la financiera está tomando protagonismo en el ámbito educativo, especialmente a partir de su inclusión en las pruebas que, cada tres años, se elaboran en el marco del programa PISA (Instituto Nacional de Evaluación Educativa, 2012: cap. 1); a pesar de ello, no es una competencia nueva, existiendo ya artículos, libros y otros documentos sobre esta temática desde la primera mitad del siglo pasado, si bien su mayor crecimiento parece haberse puesto de manifiesto, en términos de producciones científicas, en fechas más recientes.

La trascendencia de la Competencia Financiera, desde el punto de vista de la educación, está siendo cada vez mayor dada la evolución y la complejidad que la Economía está teniendo en la historia reciente. En 2006 el Congreso de los Estados Unidos, preocupado por el nivel de competencia financiera de sus ciudadanos, crea la Comisión de Educación y Alfabetización Financiera mediante la aprobación de la Ley de Mejoramiento de la Educación y Alfabetización Financiera, ya que reconoce que la complejidad de los mercados financieros es cada vez mayor y los consumidores deben disponer del conocimiento, información y habilidades necesarias para evaluar sus opciones y poder tomar decisiones que se adapten a sus necesidades y circunstancias (Financial Literacy and Education Commission (U.S.), 2006: v). La OCDE no restringe el ámbito de actuación de la Competencia Financiera solo al aspecto financiero, y destaca su relevancia desde la propia definición (Figura 1), en la que, dentro de las finalidades de la competencia, destaca que la actuación consciente y responsable de los ciudadanos no solo mejora su bienestar sino también el de la sociedad.

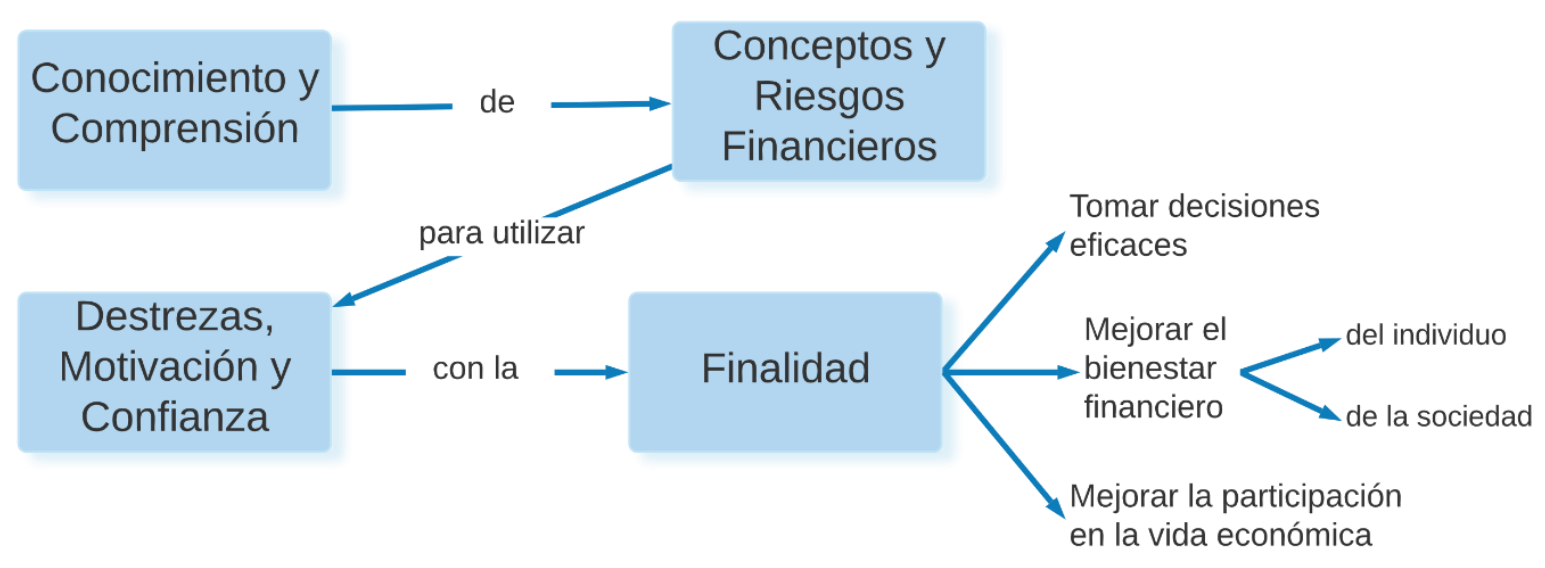

Figura 1 Concepto de Competencia Financiera. Fuente: (OECD, 2013: 144)

En un sentido diferente, Björklund y Sandahl (2020: 5) proponen superar el modelo de la OCDE, al que critican por asignar toda la responsabilidad financiera al individuo y no fomentar la revisión crítica del propio sistema financiero; las Ciencias Sociales en el sistema educativo sueco proponen una concepción de ciudadanía más amplia que la mera actuación dentro del sistema político actual, por lo que la educación financiera debe servir para fomentar una ciudadanía responsable, participativa y orientada a la justicia social (Westheimer \& Kahne, 2004: 3-4). 
La inclusión, tanto en la educación formal como informal, de programas y propuestas para trabajar la competencia financiera debe partir, como todo cambio curricular, de evidencias, las cuales deben orientar no solo la toma de decisiones inicial sino también el seguimiento y la evaluación del impacto de las acciones llevadas a cabo. En este sentido, las evidencias que proporciona la investigación y, en particular, la investigación educativa, resultan clave. La búsqueda de tales evidencias debe tomar en consideración marcos, paradigmas dominantes y múltiples fuentes y documentos, por lo que suele ser recomendable iniciar el proceso a través de rigurosos procesos de revisión de la literatura.

La revisión de la literatura, de hecho, se ha considerado como uno de los pilares fundamentales a la hora de llevar a cabo un proceso de investigación, tanto en lo que se refiere a la determinación del marco conceptual del que se va a partir, como para el análisis de la metodología, los métodos de investigación o las herramientas de análisis a emplear. En particular, Hart (1998: 13) define la revisión de la literatura como "La selección de documentos disponibles (tanto publicados como inéditos) sobre el tema, que contienen información, ideas, datos y evidencias escritas desde un punto de vista particular para cumplir con ciertos objetivos o expresar ciertas opiniones sobre la naturaleza del tema y cómo se va a investigar, y la evaluación efectiva de estos documentos en relación con la investigación propuesta".

En el caso que nos ocupa, se pretende llevar a cabo una revisión de la literatura dentro de la esfera educativa, revisión orientada a analizar las particularidades de los estudios publicados, así como las tendencias de las nuevas aportaciones y teorías aplicables en la educación (Ramírez-Montoya \& Lugo-Ocando, 2020: 17). Así, si bien el presente artículo lleva a cabo una revisión de la literatura centrada inicialmente en todos los ámbitos de la Competencia Financiera, centra la atención en todo aquello concerniente a la educación. De modo más explícito, se plantea la realización de un análisis sistemático de la literatura cuya finalidad sea identificar los artículos publicados que hagan referencia a los conceptos de Competencia Financiera y Alfabetización Financiera, delimitar los ámbitos en que se desarrollan dichos trabajos de investigación, los tópicos sobre los que trabajan y cómo han ido evolucionando a lo largo del tiempo.

\section{MÉTODO}

Se considera apropiada la realización de un Mapeo Sistemático (Sánchez Reynoso \& Diván, 2020: 22) cuando el tema de investigación es muy amplio, si se pretende revisar la literatura existente hasta este momento (Li et al., 2015: 193-194) y si el objetivo es determinar las evidencias que la literatura ha destacado hasta este momento (Kosar et al., 2016: 78). Todo ello lleva a plantear la utilización de un Mapeo Sistemático para revisar la literatura en torno a los conceptos de "Competencia Financiera" y "Alfabetización Financiera".

La revisión va a incluir ambos conceptos sin exclusión de ningún artículo, independientemente de cuál sea el ámbito científico en el que se enfoquen las publicaciones.

\subsection{Preguntas de investigación}

El objetivo de la revisión será el análisis de todos los estudios primarios publicados en revistas de ámbito científico para obtener una visión integral de los conceptos de Competencia Financiera y Alfabetización Financiera, una visión general del estado actual de las investigaciones en torno a dichos conceptos y de su evolución en el contexto de las investigaciones educativas. 
Para alcanzar los objetivos se plantean las siguientes preguntas de investigación:

¿Cómo está evolucionando la investigación sobre la Competencia Financiera y la Alfabetización Financiera? ¿Qué países lideran la publicación de trabajos?

¿Qué líneas de trabajo se están confirmando?

¿Cómo ha evolucionado la literatura en el ámbito educativo? ¿Qué dimensiones están centrando la publicación de estudios educativos sobre la Competencia Financiera y la Alfabetización Financiera?

\subsection{Búsqueda}

Habiendo fijado en los párrafos precedentes el objetivo y, dado que no se van a emplear varios revisores, se establece como criterio de búsqueda la exploración de la base de datos de referencias bibliográficas y citas Scopus, herramienta que garantizará la amplitud de la búsqueda, utilizando aquellos que valoren en primer lugar la relevancia del artículo y en segundo el número de citaciones (Rovira, Codina, GuerreroSolé, \& Lopezosa, 2019: 5). La elección de la base de datos bibliográfica Scopus, frente a otras opciones como Web of Science o Google Scholar, se ha tomado no solo por ser la de mayor amplitud, o por su revisión por pares, sino también, y de una manera especial, por la clasificación temática de las publicaciones de su repositorio; aunque Web of Science también organiza las publicaciones por ámbito temático al que pertenecen, su sistema de clasificación es menos refinado que el de Scopus, tal y como se puede apreciar en la Figura 2 al comparar la organización temática de las publicaciones si se realiza en ambas bases de datos la misma consulta sobre el tópico Financial Competence.
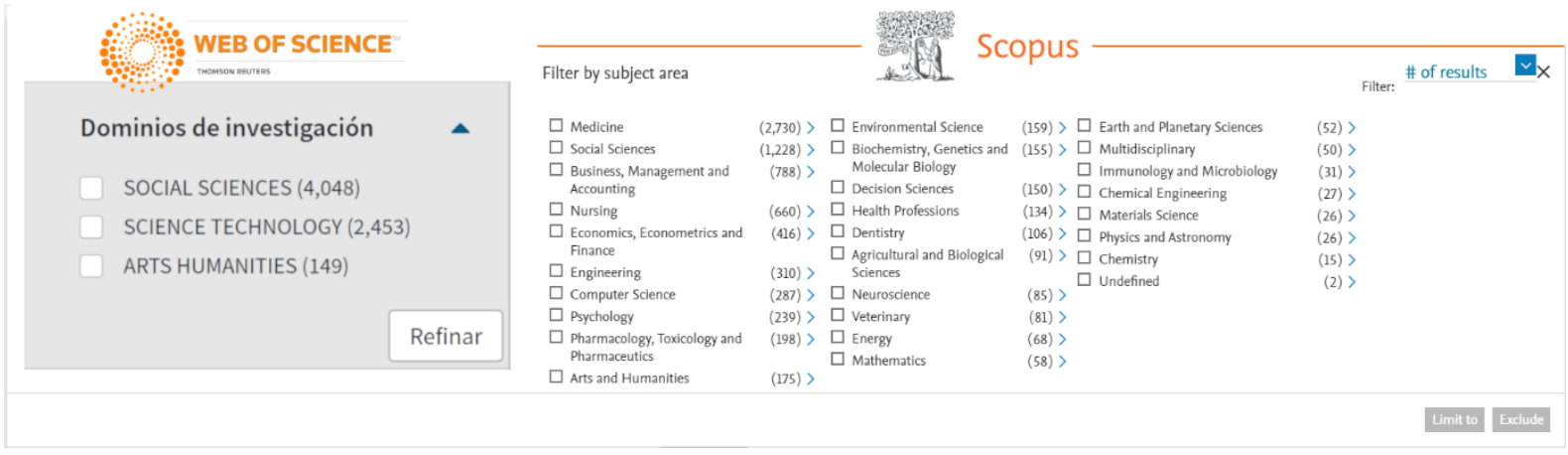

Figura 2 Comparativa de la organización temática en Web of Science y Scopus.

Como se puede ver, Scopus no solo recoge casi un $25 \%$ más de publicaciones, sino que su sistema de clasificación temático es mucho más minucioso, llegando a los 28 ámbitos temáticos frente a los 3 de Web of Science.

En los últimos años se tiende a utilizar en la literatura científica el concepto de Financial Literacy en un sentido análogo al de Financial Competence; en esta línea, el programa PISA, al diseñar el marco para realizar la evaluación financiera en 2021 (OCDE, 2019: 18), va a emplear el concepto de Financial Literacy con el mismo alcance que tenía Financial Competence en las evaluaciones anteriores. Se van a incluir ambos conceptos como objeto de la búsqueda al considerar que se están utilizando con una orientación semejante en el ámbito de la investigación en Educación Financiera. Por lo que 
respecta al término Financial Literacy, la búsqueda en ambas bases de datos bibliográficas arroja unos resultados prácticamente iguales, reportando en Web of Science un $2 \%$ más de artículos que en Scopus; a pesar de esta leve diferencia, se seguirá considerando la utilización de Scopus por su superioridad en el número de resultados del constructo "Financial Competence" y por la mejor clasificación de los artículos por ámbito científico al que pertenecen.

A la hora de realizar la consulta en la base de datos bibliográfica Scopus se ha considerado oportuno refinar los criterios de búsqueda para tener en cuenta aspectos pragmáticos y de calidad (Grant \& Booth, 2009: 97); se ha optado por utilizar la opción de "búsqueda por frase exacta", dado que al hacer una consulta simple incluyendo dos conceptos los resultados obtenidos no eran adecuados para documentar los antecedentes de la presente investigación ni por el volumen de resultados obtenidos, que abarcaban 5.785 resultados de Competencia Financiera y 3.839 de Alfabetización Financiera, ni por la temática de dichos artículos, que en unas ocasiones solo hacen referencia a uno de los dos conceptos al tiempo que en otras ocasiones incluyen los dos conceptos pero no de manera conjunta. El aspecto pragmático ha supuesto que solo se incluyeran publicaciones realizadas en el idioma inglés debido a que la búsqueda por frase exacta en francés y español no produjo ningún resultado, descartando, por tanto, el resto de los idiomas.

Se asume que la asunción de los criterios de búsqueda anteriormente descritos pueden suponer la aparición de sesgos (Beltrán \& Óscar, 2005: 64) como la existencia de publicaciones no incluidas en Scopus o que no incluyan las palabras clave especificadas en la búsqueda por frase exacta, aunque también se han evitado otros sesgos como el de publicación múltiple.

\subsection{Evaluación}

Grant \& Booth (2009: 95) argumentan que en las Revisiones de Mapeo de la Literatura no se realiza una evaluación formal de la calidad de las publicaciones analizadas. Tampoco se realizará en este caso ya que, como se ha especificado anteriormente, el objetivo del mapeo es obtener una visión integral y no responder a una pregunta de investigación concreta sobre un tema, en cuyo caso se plantearía la realización de una revisión sistemática (Manterola, Astudillo, Arias, \& Claros, 2013; Xiao \& Watson, 2019: 3).

El objetivo de un Mapeo Sistematizado de la literatura es realizar la descripción de un campo de investigación (Grant \& Booth, 2009: 97), que se encuadra como uno de los propósitos de las investigaciones (Runeson \& Höst, 2009: 134), por lo que una vez depurados los criterios de búsqueda, todas las publicaciones resultantes se incluirán en la revisión.

\subsection{Síntesis y Análisis}

Teniendo presente que una revisión sistemática de la literatura no se debe entender como una clasificación, las siguientes fases se realizarán desde un punto de vista cualitativo (Hart, 1998: 16). Así, una vez realizada la búsqueda en las bases de datos bibliográficas y recogidos todos los artículos que formarán parte de la revisión de la literatura, las fases de síntesis y análisis se realizarán conjuntamente. En este sentido, Codina (2018: 23) recoge que, habitualmente, la síntesis suele ser narrativa y que se acompaña de tablas y diagramas, aspecto que también recogen Grant y Booth (2009: 94) en esta misma etapa. La fase de análisis, por su parte, debe ser descriptiva y hacer una valoración global de los resultados, según presenta el Modelo SALSA (García Peñalvo, 2017: 23-24), aunque puede depender de la calidad y cantidad de literatura 
incluida en la revisión (Grant \& Booth, 2009: 94). Codina (2018: 22), dentro del marco de trabajo ReSiste-CHS, recoge la idea central de las revisiones sistematizadas y del mapeo sistemático de la literatura para la fase de análisis (Kosar et al., 2016; Petersen et al., 2015; Salvo et al., 2018; Sánchez Reynoso \& Diván, 2020) cuando afirma que el análisis tiene que ser un "procedimiento sistemático que asegure que cada artículo o informe ha sido tratado de forma similar".

La síntesis y el análisis se llevarán, por tanto, de forma simultánea, analizando las variables que se vayan identificando por medio del software de mapeo bibliométrico VOSviewer (van Eck \& Waltman, 2021) o por medio de tablas de datos, de manera que los datos de todas las publicaciones se analizarán y serán presentados por medio de gráficos elaborados con los mismos criterios.

De las diversas herramientas informáticas que permiten la realización de análisis bibliométricos por medio de la representación de mapas y redes bibliométricas, se ha optado por VOSviewer (van Eck \& Waltman, 2010). El hecho de que sea software libre, que pueda generar varios tipos de mapas diferentes, que presente la fuerza de la relación de dos elementos mediante mapas basados en distancias, que agrupe las muestras relacionadas entre sí por medio de clústeres o que, más específicamente respecto a las demás aplicaciones, sea capaz de trabajar con grandes volúmenes de referencias, han sido las características relevantes que han llevado a la elección de vOSviewer para la realización del análisis bibliométrico.

A partir de los datos recogidos en las búsquedas bibliográficas realizadas en Scopus, se utilizará VOSviewer para crear los mapas bibliométricos utilizando la opción de realizar un análisis de co-ocurrencia respecto a todos los conceptos o palabras clave que definen los aspectos más relevantes tratados en el contenido de los artículos. Se han elegido la visualización por superposición para que el gráfico resultante identifique, por medio de un código de colores, la franja temporal en que los artículos a los que hacen referencia las palabras clave se han publicado, y la visualización en red, que organiza los conceptos por clústeres, tanto más próximos entre sí cuanto mayor sea la relación entre ellos, y a los que asigna un color que permita identificar las agrupaciones temáticas con facilidad.

\section{RESULTADOS}

Como se puede observar en la Tabla 1, la consulta relativa a artículos cuyo título, resumen o palabras claves se refieran estrictamente a Competencia Financiera y a Alfabetización Financiera (búsqueda de frase exacta), devuelve unos resultados que muestran la publicación de pocos artículos durante medio siglo, pero cada quinquenio del s. XXI aumenta significativamente el número de publicaciones, incremento que especialmente notable desde el año 2015 en el caso de la Alfabetización Financiera.

Tabla 1 Artículos registrados en Scopus por año de publicación

\begin{tabular}{|r|r|r|r|}
\hline & $\begin{array}{c}\text { Competencia } \\
\text { Financiera }\end{array}$ & $\begin{array}{c}\text { Alfabetización } \\
\text { Financiera }\end{array}$ \\
\hline $1944-2000$ & 7 & $1984-2000$ & 10 \\
\hline $1944-2005$ & 14 & $1984-2005$ & 49 \\
\hline $1944-2010$ & 23 & $1984-2010$ & 185 \\
\hline $1944-2015$ & 38 & $1984-2015$ & 696 \\
\hline $1944-2021$ & 72 & $1984-2021$ & 2.318 \\
\hline
\end{tabular}

educade, no 12,2021, p. 8 
La utilización de la base de datos bibliográfica Scopus, también ha permitido obtener datos de los ámbitos temáticos a los que se refieren los artículos filtrados. Tomando como base los resultados obtenidos de las búsquedas realizadas en Scopus, y recogiendo la distribución por ámbitos temáticos de cada listado de artículos, se ha elaborado la Tabla 2. El período temporal considerado para recoger los datos de publicación por ámbito temático ha sido desde la fecha de publicación del primer artículo hasta la actualidad.

Como se puede apreciar en una primera aproximación, el ámbito de Ciencias Sociales es el que destaca en ambos conceptos al presentar un porcentaje elevado y bastante similar en las búsquedas en ambos conceptos.

En el caso de la Competencia Financiera, los artículos del ámbito de la Psicología y la Medicina (10,2\% y $18,1 \%$ respectivamente) tienden a estudiar la capacidad de obrar de las personas en situaciones en que cabe duda de que puedan ejercer adecuadamente dicha facultad, bien sea por edad (ancianos y jóvenes menores de edad) o por enfermedad, aunque algunos estudios tienen por finalidad establecer herramientas de valoración de la capacidad financiera para responder a demandas de peritaciones realizadas por las autoridades sanitarias o judiciales.

En el concepto de Financial Literacy destacan las publicaciones relacionadas con la Economía (Economía, Econometría y Finanzas un $27,6 \%$ de las publicaciones y Negocios y Gestión de Cuentas con un $20,3 \%$ ) y con las Ciencias Sociales $(23,3 \%)$, que acaparan en total más del $71 \%$ de las publicaciones totales, siendo el resto de los ámbitos minoritarios y no superando en ningún caso el $5 \%$. La utilización del concepto Financial Competence también se recoge en las disciplinas de los ámbitos económico (Economía, Econometría y Finanzas un 16,5\% de las publicaciones y Negocios y Gestión de Cuentas con un $13,4 \%$ ) y de las Ciencias Sociales $(21,3 \%)$, pero también tienen un peso específico significativo dos disciplinas bastante alejadas de éstos: la Medicina, que alcanza un porcentaje de publicación del 18,1\%, y la Psicología, con el 10,2\%.

Es destacable que el $48,8 \%$ de las publicaciones relacionadas con el concepto Financial Competence no están relacionadas con los ámbitos económicos o con las Ciencias Sociales y que, dentro de este grupo, hay dos ámbitos que acaparan el $28,3 \%$ del total, llegando incluso la Medicina a ser el segundo mayor ámbito de publicación. Por su parte, respecto a la Financial Literacy, el peso de las ciencias no relacionadas con la Economía o las Ciencias Sociales solo llega al 28,8\%, no destacando ningún ámbito en particular, ya que ninguno supera el $5 \%$. 
Tabla 2 Relación de publicaciones sobre la Competencia Financiera según el ámbito temático

\begin{tabular}{|c|c|c|}
\hline & $\begin{array}{l}\text { Competencia } \\
\text { Financiera }\end{array}$ & $\begin{array}{c}\text { Financial } \\
\text { Literacy }\end{array}$ \\
\hline Social Sciences & $21,3 \%$ & $23,3 \%$ \\
\hline Psychology & $10,2 \%$ & $5,0 \%$ \\
\hline Mathematics & $0,8 \%$ & $1,7 \%$ \\
\hline Computer Science & $4,7 \%$ & $4,1 \%$ \\
\hline Engineering & $2,4 \%$ & $3,0 \%$ \\
\hline Arts and Humanities & $1,6 \%$ & $2,6 \%$ \\
\hline Neuroscience & $1,6 \%$ & $0,1 \%$ \\
\hline Medicine & $18,1 \%$ & $4,3 \%$ \\
\hline Physics and Astronomy & & $0,2 \%$ \\
\hline Agricultural and Biological Sciences & $1,6 \%$ & $0,8 \%$ \\
\hline Energy & & $1,2 \%$ \\
\hline Biochemistry, Genetics and Molecular Biology & $0,8 \%$ & $0,6 \%$ \\
\hline Business, Management and Accounting & $13,4 \%$ & $20,3 \%$ \\
\hline Chemical Engineering & & $0,1 \%$ \\
\hline Health Professions & $1,6 \%$ & $0,2 \%$ \\
\hline Multidisciplinary & & $0,4 \%$ \\
\hline Earth and Planetary Sciences & & $0,3 \%$ \\
\hline Materials Science & $0,8 \%$ & $0,2 \%$ \\
\hline Decision Sciences & $1,6 \%$ & $1,2 \%$ \\
\hline Environmental Science & $0,8 \%$ & $1,9 \%$ \\
\hline Nursing & $2,4 \%$ & $0,5 \%$ \\
\hline Chemistry & & $0,0 \%$ \\
\hline Economics, Econometrics and Finance & $16,5 \%$ & $27,6 \%$ \\
\hline Pharmacology, Toxicology and Pharmaceutics & & $0,3 \%$ \\
\hline Immunology and Microbiology & & $0,0 \%$ \\
\hline Veterinary & & $0,1 \%$ \\
\hline
\end{tabular}

A partir de la información obtenida de las búsquedas en la base de datos bibliográfica Scopus, se ha realizado un análisis por medio de mapas bibliométricos. Aunque, siguiendo las conclusiones de van Eck y Waltman (2014: 28), hay que tener presente que se pueden producir pérdidas de información tanto al transformar las referencias bibliográficas en redes bibliométricas como al representar gráficamente la información.

Como puede observarse en la Figura 3, en la Figura 5 y en la Figura 7, las referencias clave que identifican el contenido de los artículos relacionados con el constructo de Competencia Financiera durante los tres primeros períodos se refieren en gran medida a ámbitos no relacionados directamente con la Educación ni con la Ciencia Económica, sino que sus objetivos están encaminados a estudiar la capacidad de obrar de los individuos y surgen de los ámbitos sanitarios (Psicología, Psiquiatría o Medicina entre otros) y pueden ser identificados en el clúster de color más oscuro correspondiente a los primeros años del período contemplado; otro de los ámbitos con mayor peso en el período elegido es el vinculado al entorno legal (valoración de situaciones o juicios en los que se busca determinar si una persona tiene capacidad para disponer libremente de sus bienes), que se pueden identificar en los clústeres restantes y que corresponden temporalmente a los años centrales y finales del período objeto de análisis.

En las publicaciones anteriores al año 2000 se puede apreciar cómo el concepto de Competencia es el centro de las publicaciones y lo que relaciona entre sí los ámbitos más conectados con el medio ambiente (en el clúster amarillo del gráfico: Eliminación 
de desechos clínicos, Incineración, Protección medioambiental, ...) y aquellos del ámbito de la salud (en los clústeres verde y morado: Toma de decisiones, Medidas legales, Ancianos, Desórdenes cognitivos, ...).

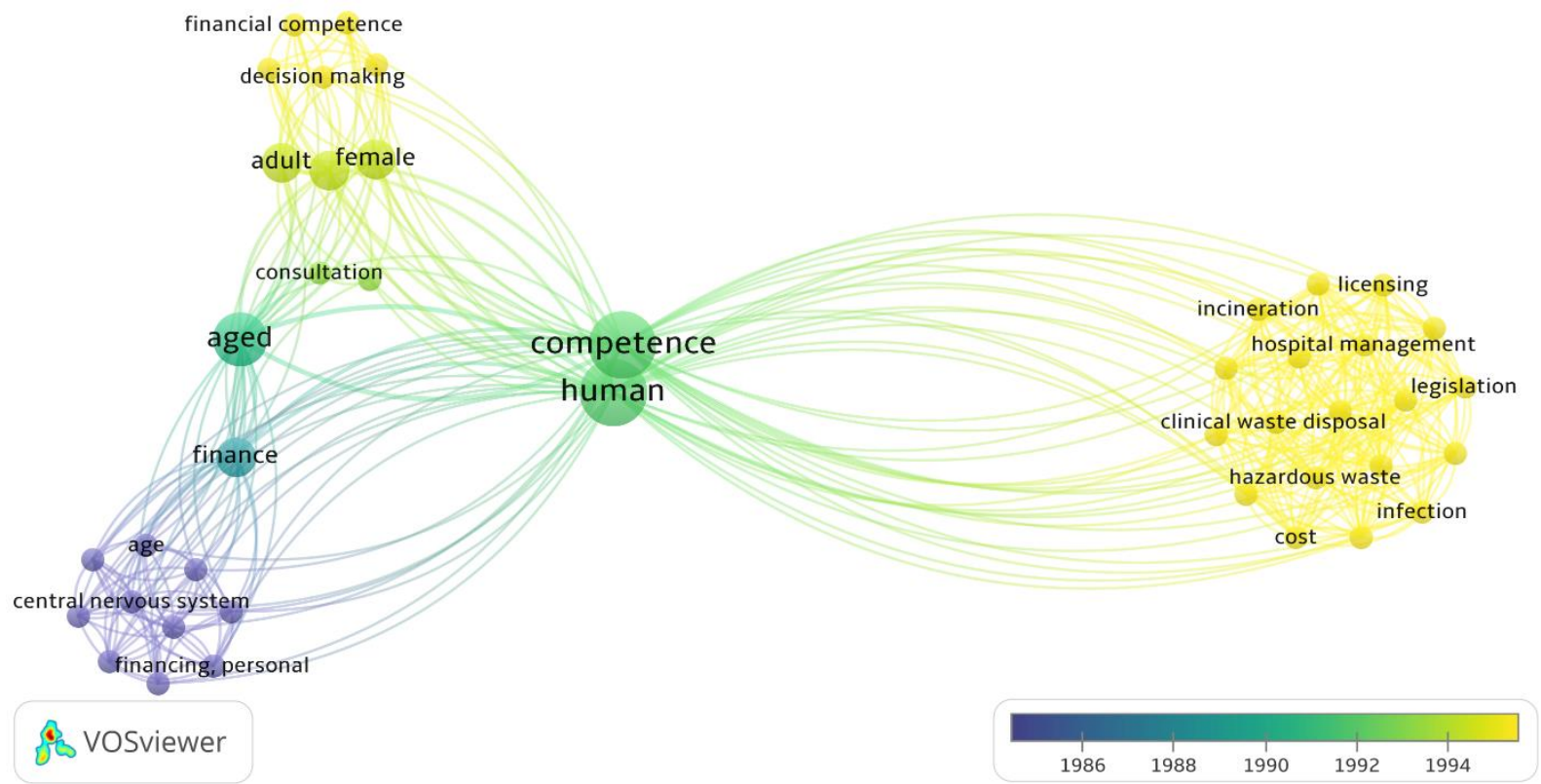

Figura 3 Competencia Financiera 1944-2000. Análisis de co-ocurrencia por conceptos clave

Los artículos relativos a la Alfabetización Financiera no se recogen hasta el año 1984 y, como se puede apreciar en la Figura 4, su ámbito de conocimiento es variado, con referencias sociales como la Inclusión financiera o de negocios o como los artículos relacionados con los Mercados emergentes o los Préstamos; la temática va evolucionando hacia temas más relacionados con la Competencia Financiera (en el clúster verde se recogen la Conciencia financiera y las Habilidades financieras entre otros conceptos) y con el ámbito educativo (el clúster amarillo recoge la Evaluación de programas, las Finanzas en los adolescentes y el Currículum financiero, temas todos ellos relacionados con la Educación).

Dado que los períodos estudiados son acumulativos, los clústeres con alto valor específico en un período van a mantenerlo, como norma general, en los siguientes períodos analizados; tal es el caso de los dos clústeres relacionados con la Competencia Financiera analizados en la Figura 3 y que, a pesar de que han cambiado de posición en la Figura 5, se puede apreciar que mantienen el código de colores asociado a la referencia temporal así como su volumen, que hace alusión a su mayor presencia respecto al resto de palabras clave. 
F. Javier Sánchez Antolín y José María Marbán Competencia financiera y educación: una aproximación desde el mapeo de la ciencia

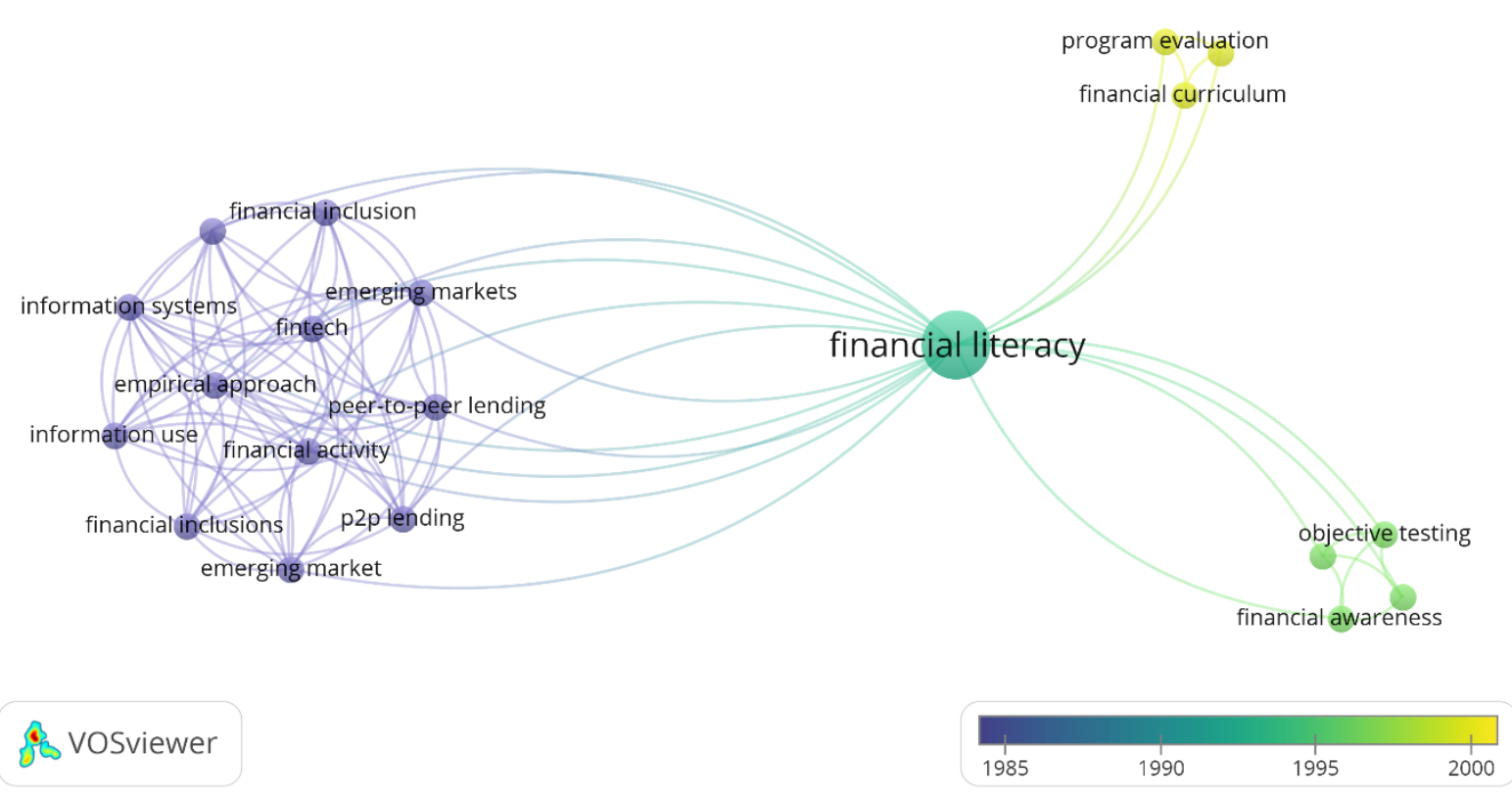

Figura 4 Alfabetización Financiera 1984-2000. Análisis de co-ocurrencia por conceptos clave

En este período se puede identificar la aparición de los primeros conceptos relacionados con diversos ámbitos de la Economía, conceptos que empiezan a tener entidad propia para destacar y para crear clústeres de palabras clave relacionadas entre sí.

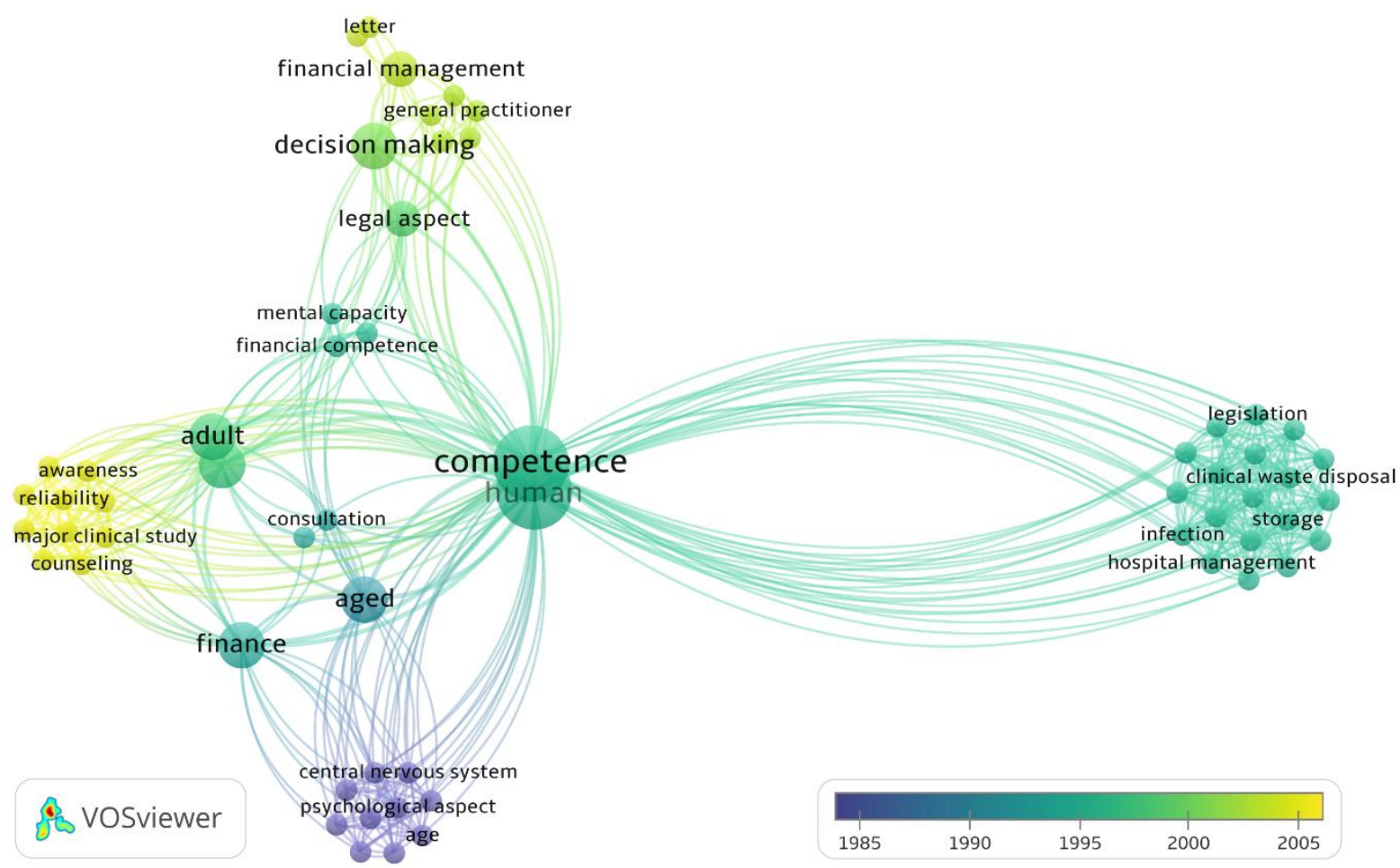

Figura 5 Competencia Financiera 1944-2005. Análisis de co-ocurrencia por conceptos clave

La evolución en la Alfabetización Financiera destaca solo en uno de los tres clústeres: la Educación. Mientras que los otros dos clústeres permanecen invariables, los temas relacionados con el ámbito educativo protagonizan un crecimiento significativo tanto 
en el número de publicaciones como en las temáticas. Ya aparece el nodo Estudiantes y dinero conectado con los Adolescentes y el Currículum, directamente relacionado con el programa PISA; surge un clúster en el que relacionan Actitudes financieras con diversos factores demográficos y temas legales, y abajo a la izquierda otro con temáticas relacionadas con las Competencias profesionales, Contabilidad, Auditoría financiera o el comportamiento social en general. Por último, encontramos en la parte superior izquierda un clúster que relaciona la Educación Financiera con diferentes Colectivos desfavorecidos y distintas Actuaciones gubernamentales.

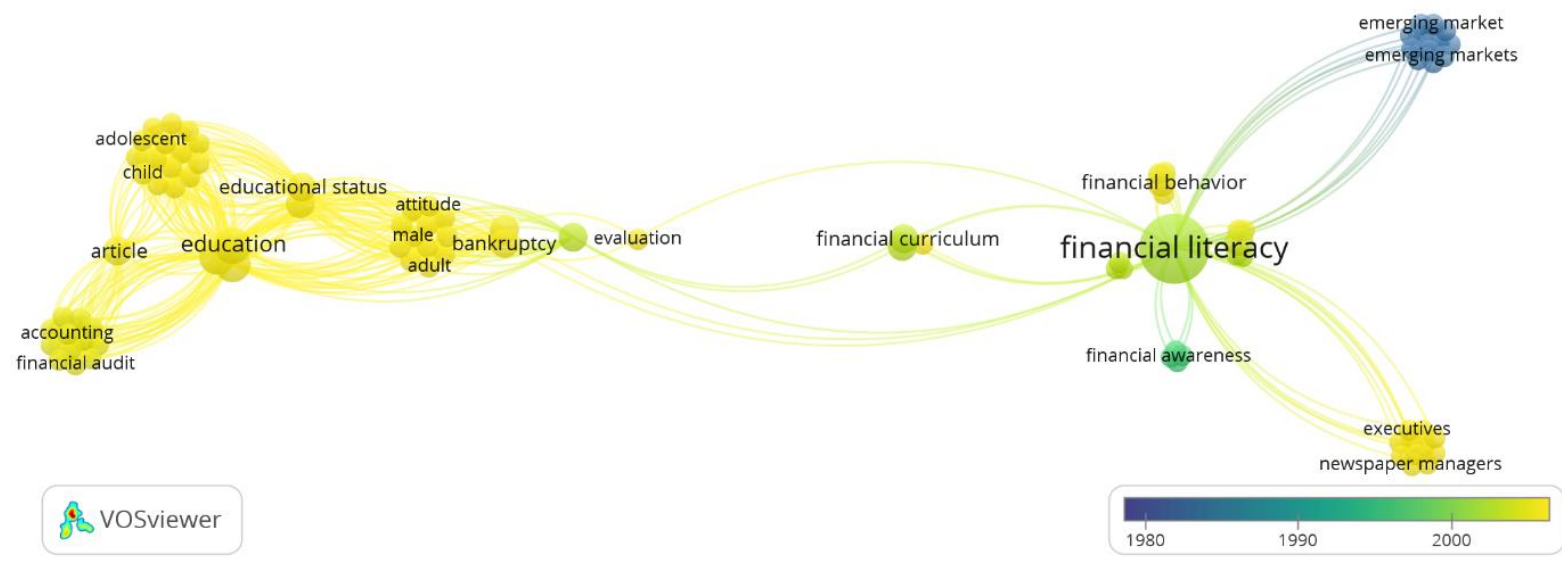

Figura 6 Alfabetización Financiera 1984-2005. Análisis de co-ocurrencia por conceptos clave

En el período 1944-2010 es destacable la distribución de la coloración en los diferentes clústeres, en los que se puede ver cómo las publicaciones más recientes sobre Competencia Financiera abarcan sustancialmente más clústeres que en los períodos anteriores. Destacan dos nuevos clústeres referidos a la Competencia Financiera y a la Evaluación, Capacidad y Toma de decisiones, que, además de mostrar una relación notable entre ellos, fácilmente identificable dada la cercanía de ambos clústeres entre sí, también destacan por el número de relaciones que tienen establecidas entre ambos. 


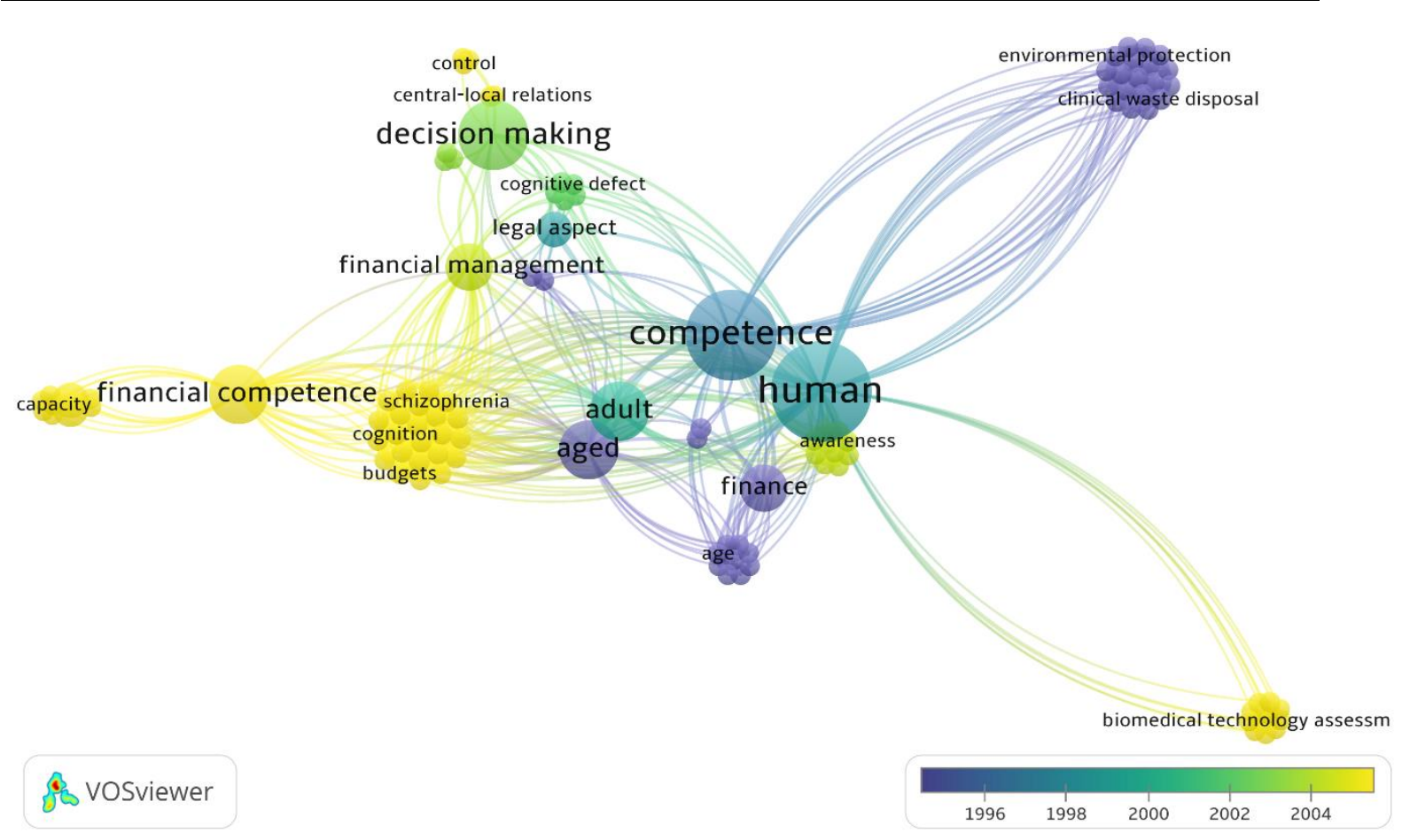

Figura 7 Competencia Financiera 1944-2010. Análisis de co-ocurrencia por conceptos clave

En este mismo período la evolución gráfica de los artículos referidos a la Alfabetización Financiera (Figura 8) es sorprendente ya que, debido al elevado número de publicaciones del último quinquenio, es prácticamente monocolor; en los últimos cinco años del período considerado se publican más del triple de artículos que en todos los años anteriores. Este impulso investigador no se ciñe a ámbitos concretos, sino que es bastante general en las temáticas investigadas, hasta el punto de que es difícil identificar grandes clústeres con la misma facilidad que en los períodos anteriores.

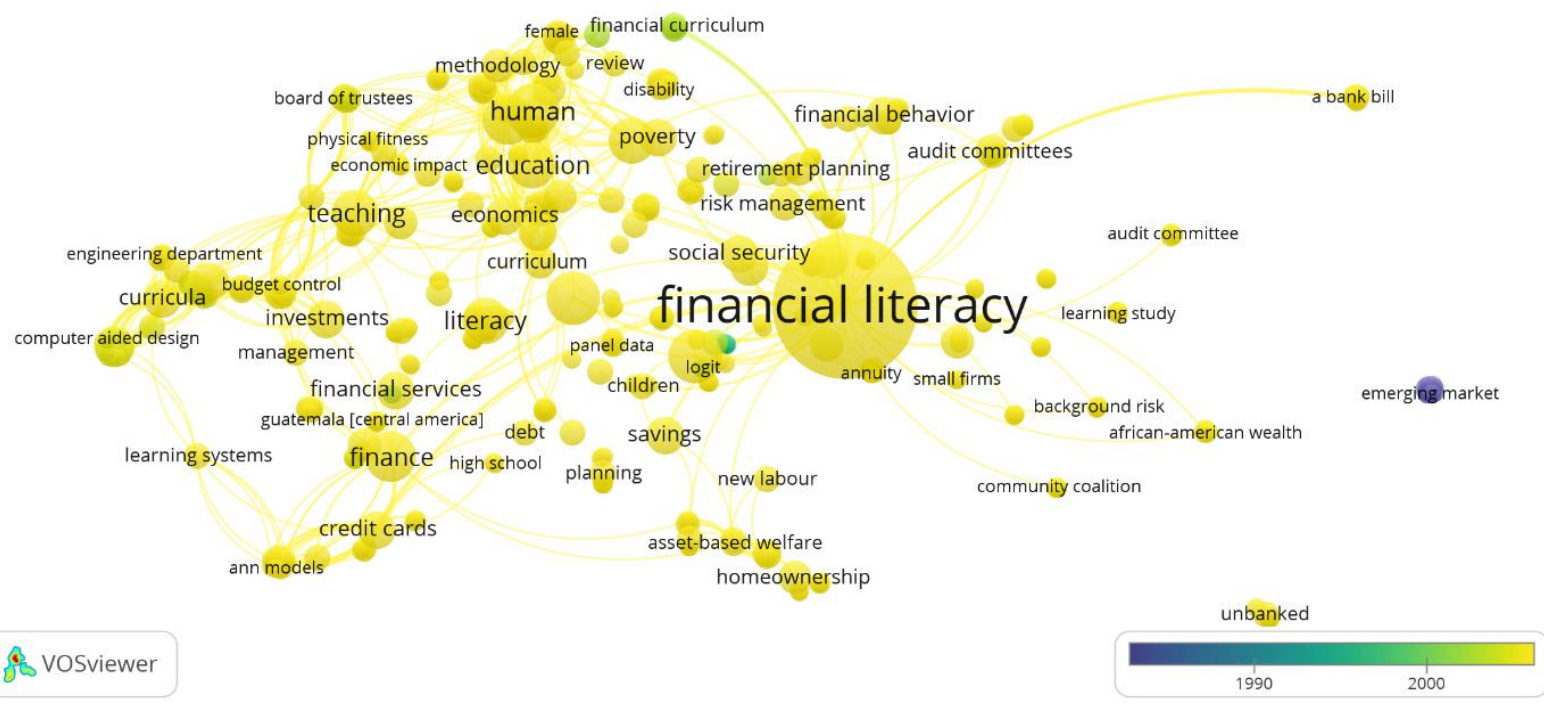

Figura 8 Alfabetización Financiera 1984-2010. Análisis de co-ocurrencia por conceptos clave 
Dos de los nodos que más destacan en este período son la Educación Financiera y las Finanzas Personales. Ambos conceptos, aunque son los que mayor volumen tienen y, por tanto, son los que mayor número de publicaciones han tenido en este período, no tienen un número elevado de relaciones con otros conceptos, como podemos observar en la Figura 9, y tampoco tienen el suficiente número de relaciones entre ellos para que aparezca dibujada en el mapa bibliométrico.

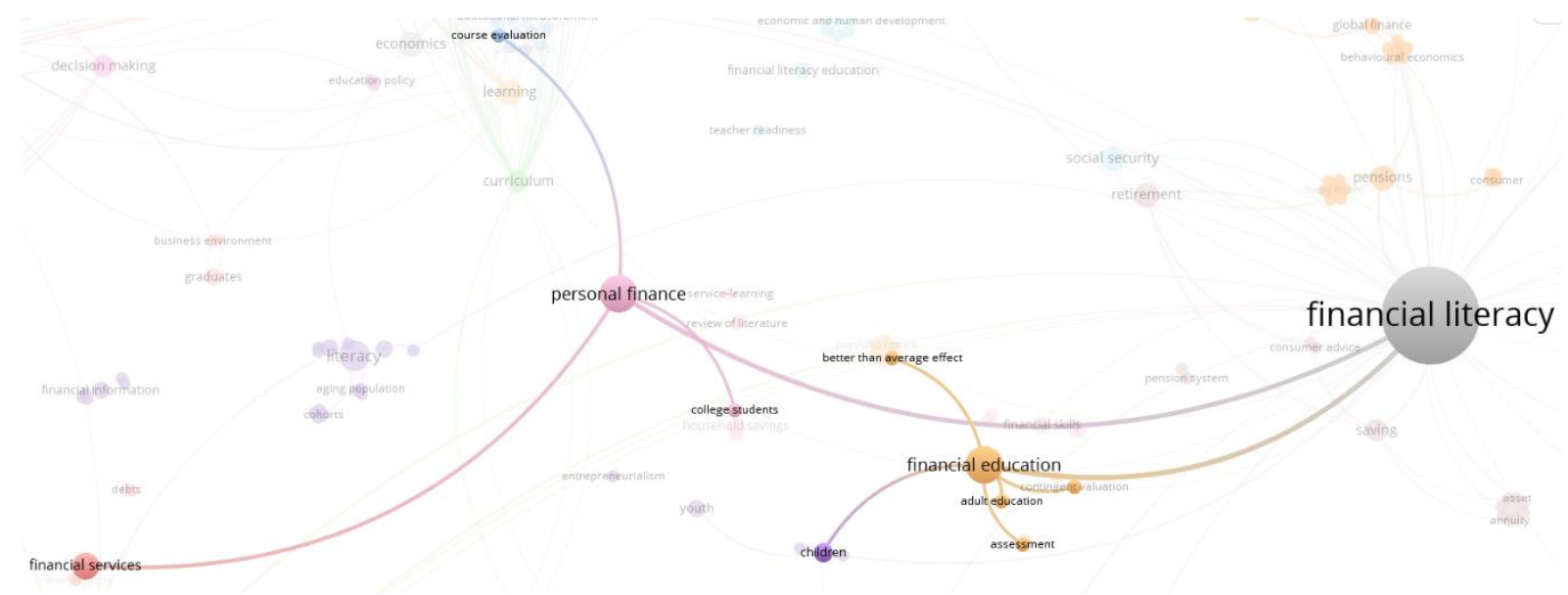

Figura 9 Alfabetización Financiera 1984-2010. Detalle de relaciones de las Finanzas Personales y la Educación Financiera

En relación con el ámbito educativo podemos identificar dos nodos que, sin ser los de mayor volumen, presentan una importancia significativa tanto desde el punto de vista de publicaciones como de relaciones con otros conceptos. Los nodos Enseñanza y Aprendizaje establecen relaciones comunes con un clúster relacionado con la Educación (marcados en color azul), y que centra muchos de sus conceptos en grupos concretos a los que va dirigida la educación (Adolescentes, Niños, Personas con discapacidad, ...), o en aspectos metodológicos (Metodología, Motivación o Comprensión), ambos también comparten la relación con el entorno de los negocios.

El nodo relativo a la Enseñanza también mantiene un grupo de relaciones destacable con dos clústeres marcados en rojo y azul claro; el clúster rojo está directamente relacionado con la enseñanza en escuelas de ingeniería y de negocios, tanto en aspectos generales como más concretos, como puede ser el Control de presupuestos. El clúster azul claro hace referencia a diversos aspectos tecnológicos como los Sistemas de información geográfica, la World wide web, los Modelos matemáticos, Estructuras de programación o Sistemas de navegación, temáticas en las que el proceso de enseñanza desarrolla la Alfabetización Financiera. 


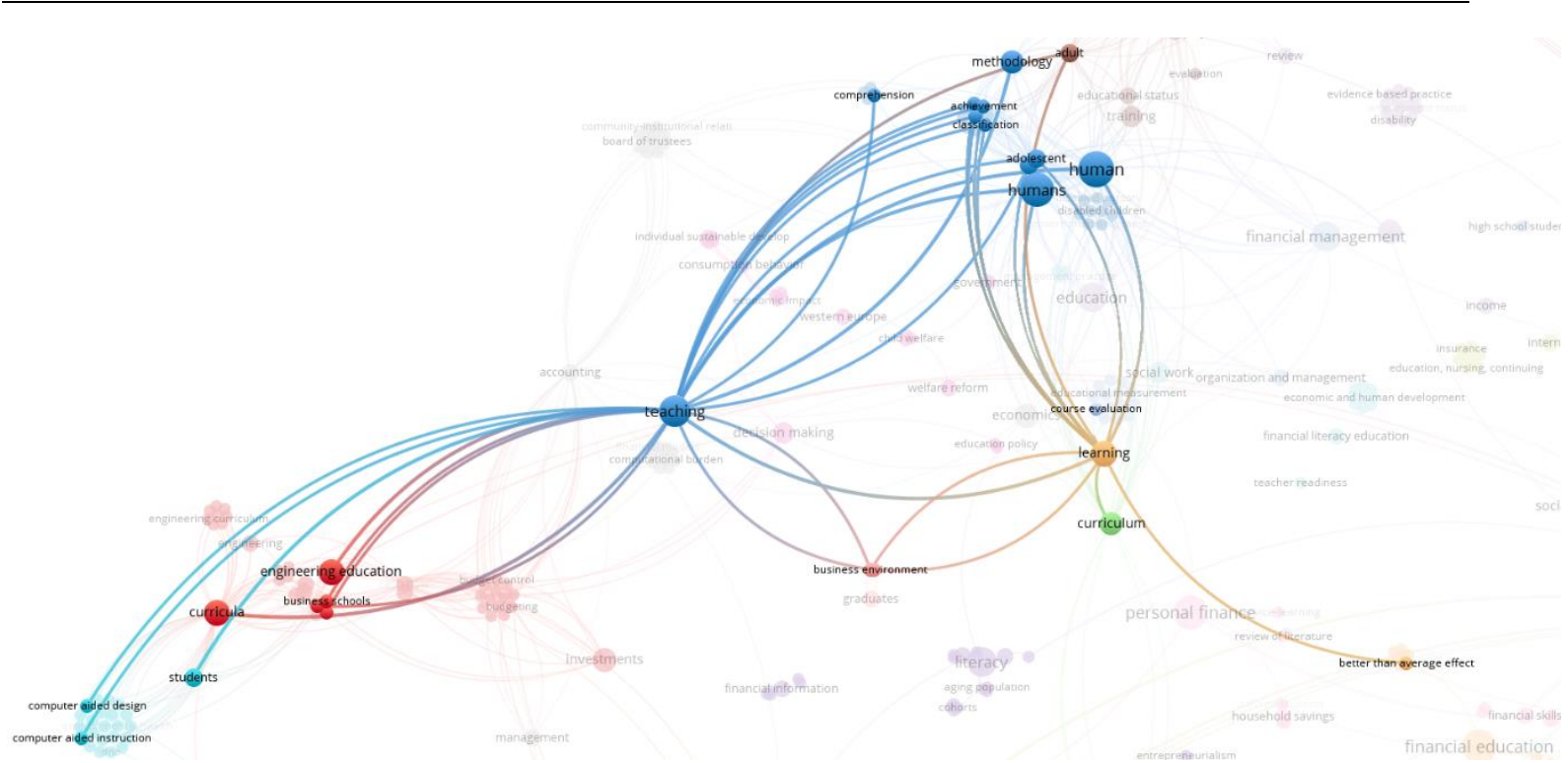

Figura 10 Alfabetización Financiera 1984-2010. Detalle de relaciones entre los conceptos de Enseñanza y Aprendizaje

En el período 1944-2015 el clúster central, si nos centramos en la Competencia Financiera, sigue siendo el relativo a Humano, pero se aprecia una mayor dispersión en cuanto a los clústeres que lo rodean. Aparecen un grupo de temáticas relacionadas con el entorno medioambiental (parte derecha de la Figura 11, en color morado), al que se adicionan un primer clúster relacionado con los Costes, y un segundo clúster en el que destacan conceptos relativos a Coste de intereses, Cadena de valor, Ventas, Producción, Expansión, Valor de mercado y Valor de accionistas.

En el otro extremo del mapa bibliométrico los clústeres relacionados con temáticas sanitarias y psicosociales aumentan el volumen de publicaciones con este tipo de palabras clave, y la relación entre ellas, ya que, como se ha explicado anteriormente, VOSviewer representa por medio de distancias las relaciones entre los ítems, situando más cerca aquellas palabras clave que tienen mayor relación entre ellas. Asimismo, se aprecia la aparición de conceptos que ganan la relevancia suficiente para destacar dentro de su clúster y marcar nuevas relaciones con otros:

- Entre los clústeres de Finanzas y Competencia Financiera, surge un grupo de nodos relacionados con la Inteligencia artificial, la Competición y con los Sistemas de evaluación comprensivos.

- La Evaluación amplía sus relaciones y pasa de relacionarse, casi exclusivamente con la Competencia Financiera, a servir de nexo entre ésta y el Envejecimiento.

- Surge un nodo relativo al Diseño y uso de cuestionarios que se relaciona con muchos de los nodos relativos a la salud física y a la situación psicosocial de las personas. Destaca el surgimiento de una temática centrada en el Análisis de grupos que se relaciona exclusivamente con el nodo de Cuestionarios.

- Cabe remarcar la evolución del nodo Finanzas, que aumenta su presencia en el mapa e incrementa el número de nodos con los que tiene relación, entre los que destacan sus relaciones con los nuevos nodos. 


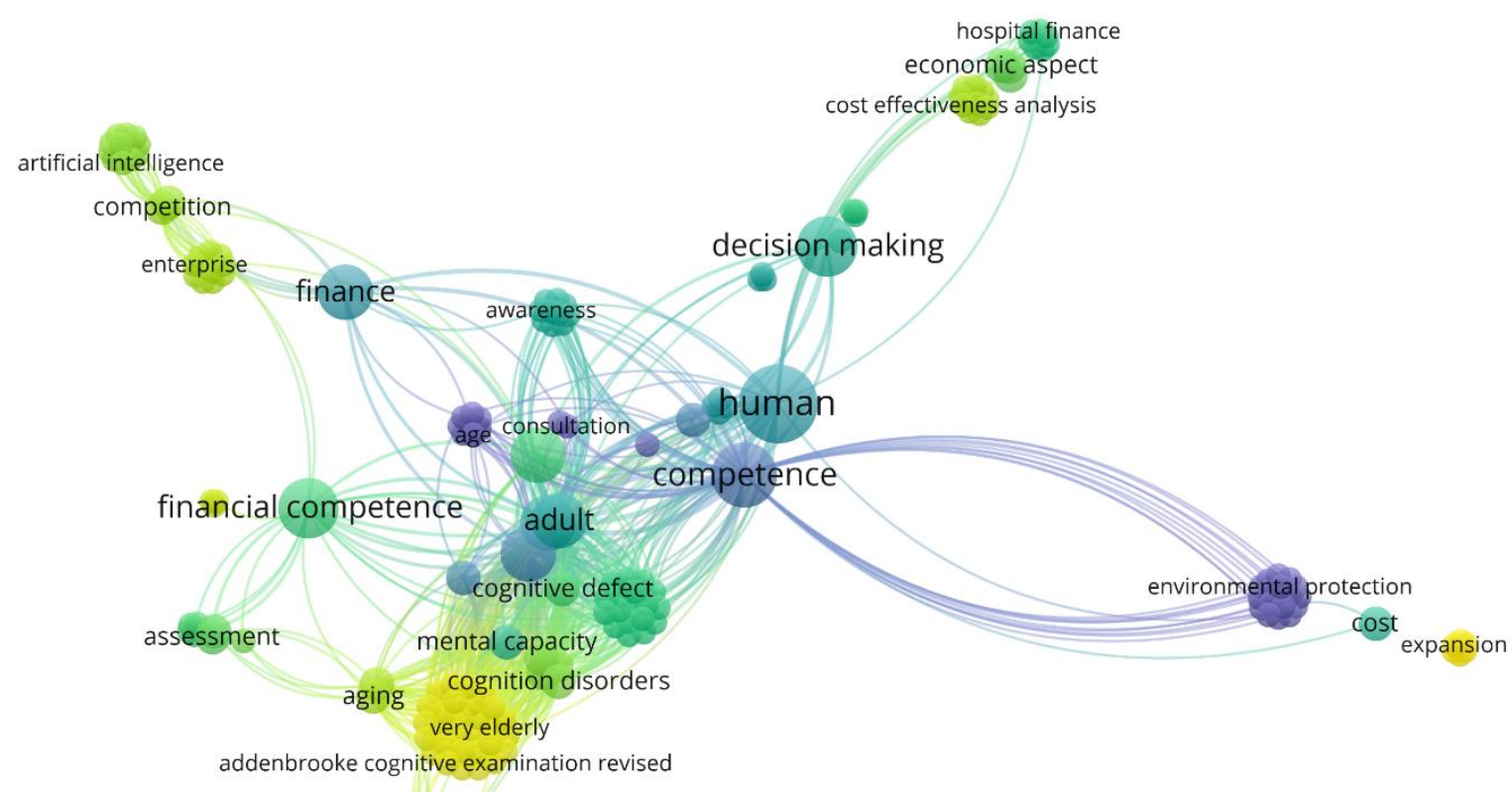

questionnaires

\& VOSviewer

cluster analysis

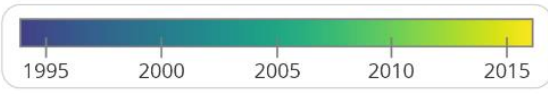

Figura 11 Competencia Financiera 1944-2015. Análisis de co-ocurrencia por conceptos clave

El mapeo de Financial Literacy presenta características similares a las del período anterior; como ya se ha reseñado anteriormente, el hecho de que en cada período considerado el número de publicaciones triplique el total de publicaciones previas hace que el mapa de análisis temporal no aporte excesiva información.

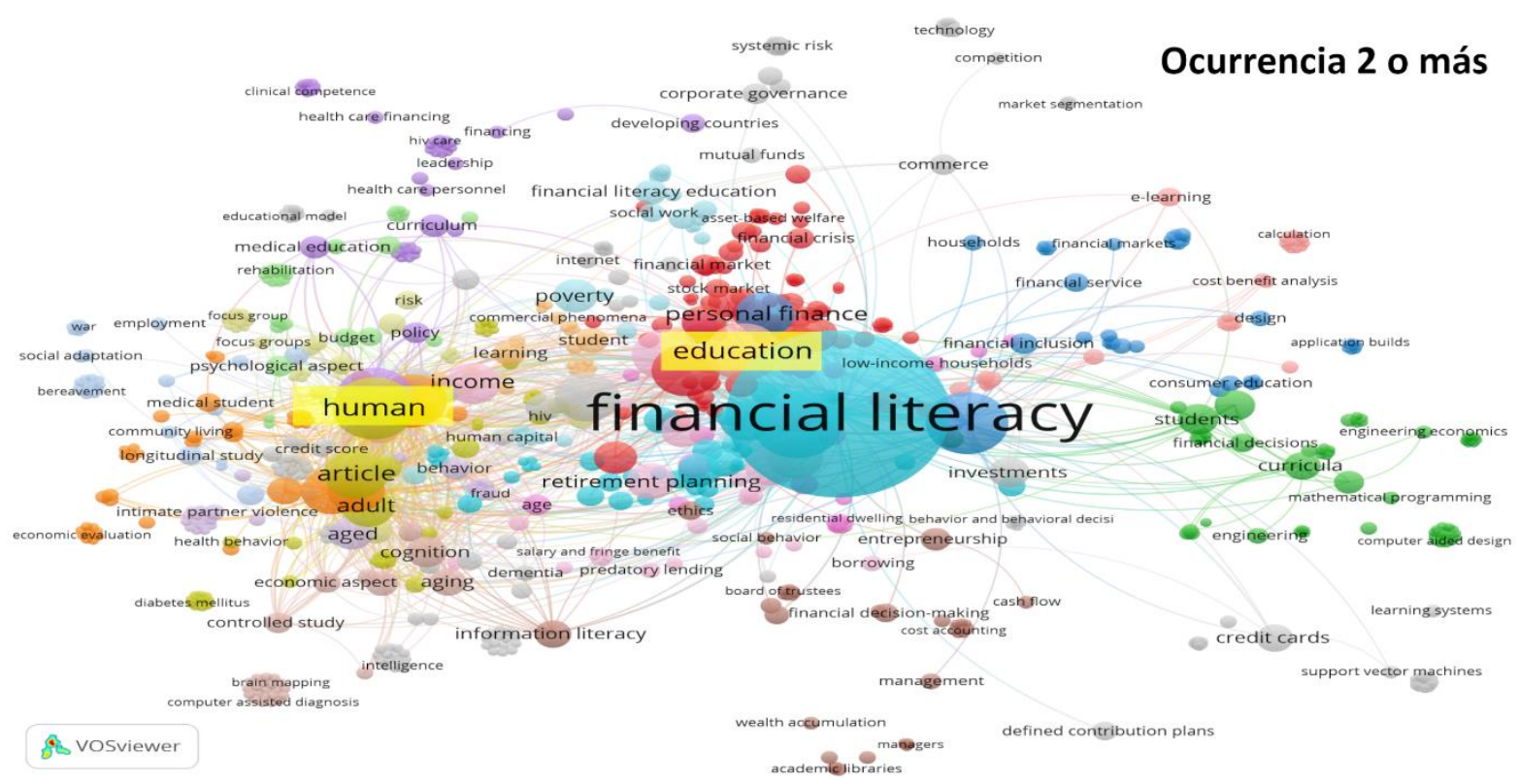

Figura 12 Alfabetización Financiera 1984-2015. Clústeres temáticos con una ocurrencia de 2 o más 
El análisis de las redes de co-ocurrencia de la Figura 12 presenta los mapas obtenidos al limitar el número de co-ocurrencias de cada palabra clave a un mínimo de 2 . En el mapa correspondiente a dos o más co-ocurrencias se percibe una variedad temática y una dispersión en los clústeres, que solapan sus conceptos y dificultan su estudio.

A medida que el criterio de co-ocurrencia se hace más estricto, se aprecia cómo los conceptos se van agrupando (Figura 13), permitiendo identificar los clústeres y las relaciones que establecen con el resto de los clústeres del mapa.

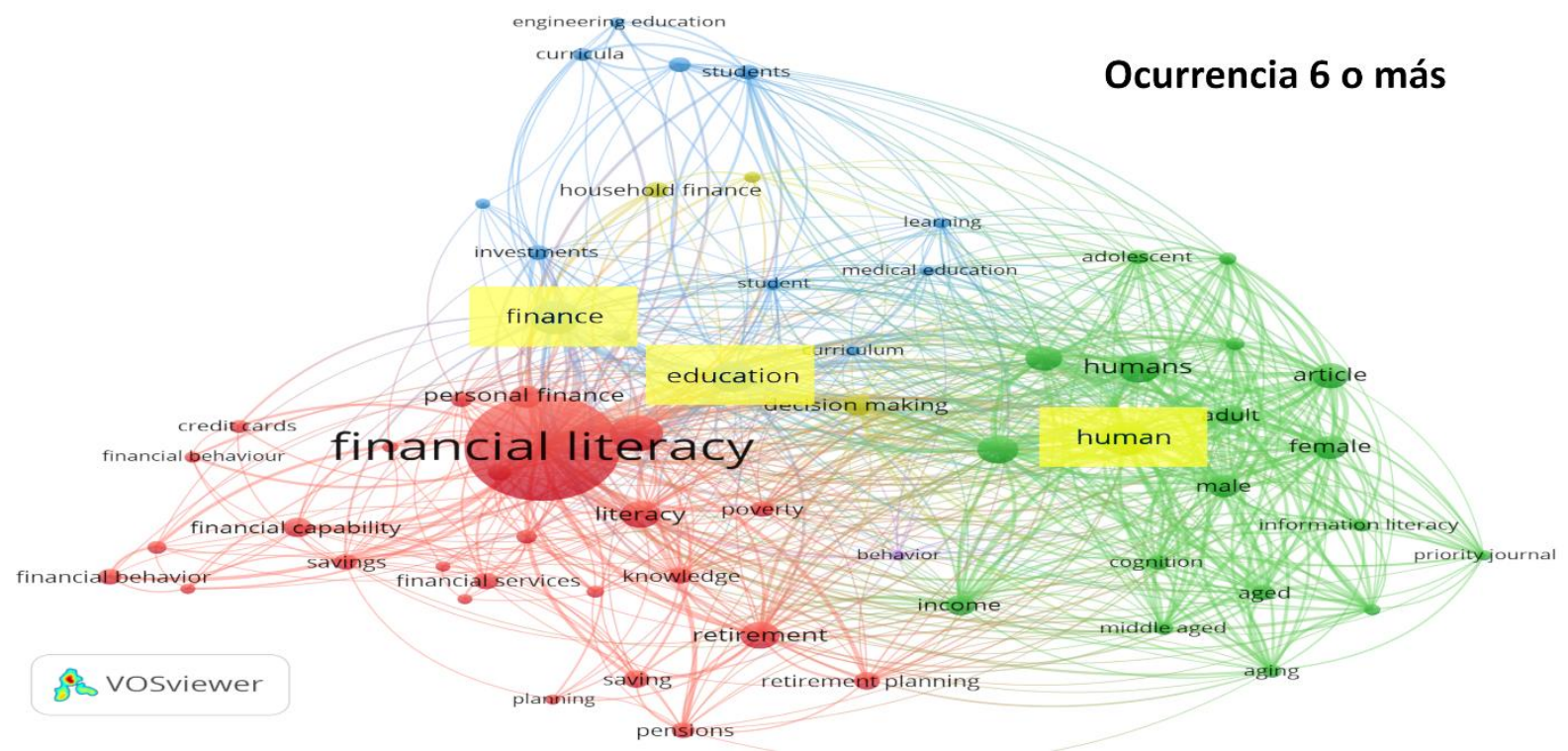

Figura 13 Alfabetización Financiera 1984-2015. Clústeres temáticos con una ocurrencia de 6 o más

La evolución de los clústeres tiende a tres temáticas diferenciadas; por un lado, el clúster azul hace referencia a situaciones concretas en las que interviene la Alfabetización Financiera, tales como el Ahorro, la Jubilación, las Pensiones, o el Análisis del comportamiento financiero. Los otros dos nodos se centran en la Educación (en el centro del mapa) y en los factores psicosociales y económicos que están directamente relacionados con la Alfabetización Financiera (clúster verde). Se observa que el clúster rojo no se identifica con claridad por falta de un referente; al aumentar el nivel de ocurrencia (Figura 14) se verá que el nodo que debido a su proximidad al concepto central no aparece es la Educación Financiera. 


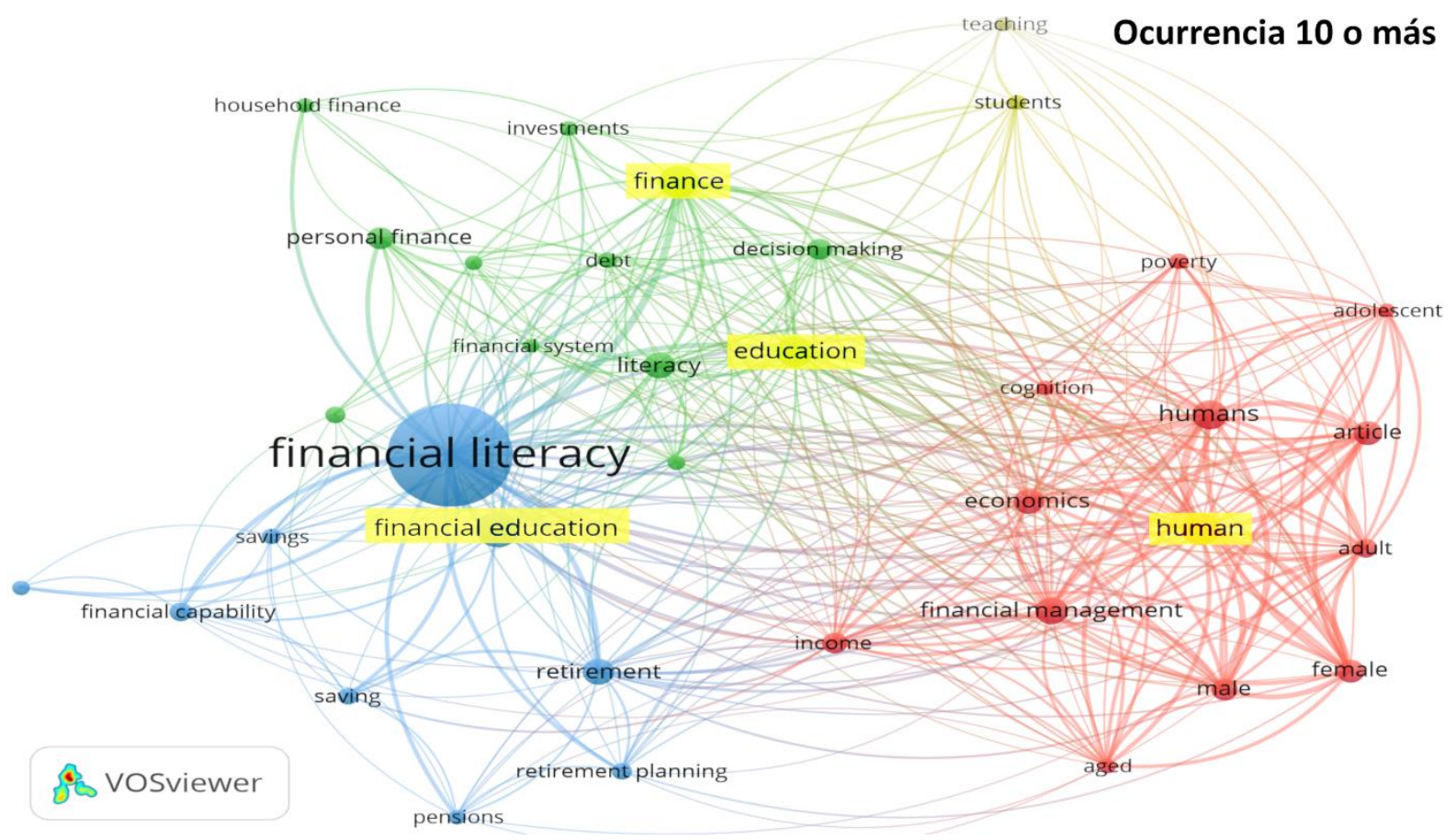

Figura 14 Alfabetización Financiera 1984-2015. Clústeres temáticos con una ocurrencia de 10 o más

Dentro de este último mapa destaca la posición de dos nodos que afianzan las fuertes relaciones existentes entre los clústeres de Educación y de Factores socioeconómicos, y que se refieren a la Enseñanza y al Papel de los estudiantes; como se puede ver en la Figura 15 estos dos nodos mantienen dobles relaciones con la mayoría de los nodos con los que se relacionan, que son aquellos con mayor volumen de ocurrencia dentro de sus propios clústeres: Educación, finanzas e inversiones en el clúster relacionado con la Docencia, y Humanos, Ciencias económicas, Adultos y Adolescentes en el clúster psicosocial y económico, todo ello aparte de la relación de estas temáticas con otras de manera individual, a las que hay que añadir la relación de las publicaciones que relacionan a los estudiantes con la Educación Financiera y a ambos nodos con la Alfabetización Financiera. 


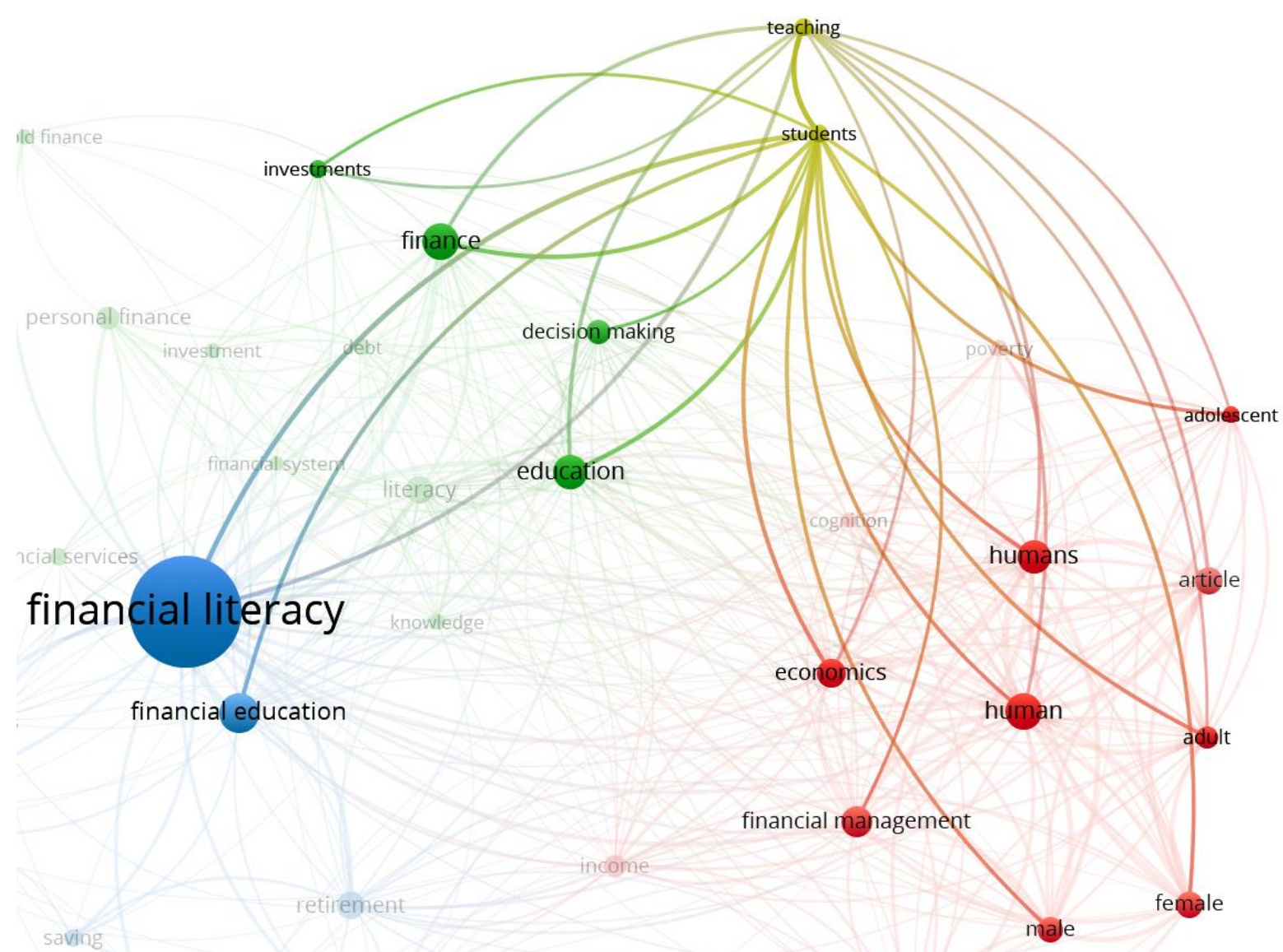

Figura 15 Alfabetización Financiera 1984-2015. Detalle de relaciones entre los conceptos de Enseñanza y Estudiantes

La simulación gráfica que incluye todas las publicaciones referidas a Competencia Financiera hasta el año 2021 muestra un desplazamiento importante de una de las ramas principales del período anterior, el refuerzo de las que eran temáticas importantes y el crecimiento y agrupación de una serie de nodos en la parte central del mapa, aunque quizás lo más destacable es la aparición de un núcleo que destaca por su volumen: la Educación Financiera; teniendo en cuenta que los informes PISA empiezan a evaluar la Competencia Financiera en 2012, es razonable pensar que este primer estudio ha podido tener una cierta influencia en la evolución de las publicaciones relacionadas con dicho núcleo temático, aunque no hay que menospreciar otras iniciativas que se han ido desarrollando sobre todo en los años posteriores, de entre las que la Autoridad Bancaria Europea recoge más de 120 en su Informe de Educación Financiera 2019-20 (European Banking Authority, 2020: Annex), o las puestas en funcionamiento por otras instituciones supranacionales, como el Proyecto de alfabetización y Educación Financiera del Banco Mundial en colaboración con el Ministerio de Finanzas de la Federación Rusa iniciado en 2008 (Mundial Bank, 2010).

Desde una perspectiva temática, se pueden identificar en el mapa relaciones que van asentando entre temáticas, y que se identifican por la cercanía de sus nodos, por el aumento de relaciones y por el crecimiento del número de publicaciones, reflejado en un mayor tamaño de la representación gráfica de dicho nodo. De entre estos casos, cabe destacar algunos que pueden ser relevantes en el presente análisis: 
Destaca el volumen de la Alfabetización financiera y el conjunto de relaciones que genera con varios grupos de clústeres, aunque sorprende la cercanía del clúster relativo a la Educación Financiera o sus conceptos relacionados (Proceso educativo, Competencias de la sociedad innovadora, Divulgación, Especializaciones académicas, ...) y que gráficamente no se aprecien relaciones entre ambos clústeres, aunque este efecto podría deberse a la, ya comentada, pérdida de información que se puede producir al traspasar datos bibliométricos para hacer el mapeo, o en el propio proceso de generación del mapa.

Los clústeres relacionados con la Alfabetización son los que conectan indirectamente los clústeres centrales (relativos a la Persona y sus Circunstancias para tomar decisiones), con el nodo relativo a la Alfabetización Financiera. Se aprecia que hay temáticas relacionadas con la Alfabetización en general, como son aspectos de Género y de Edad que también originan relaciones con la Alfabetización Financiera.

En la parte superior del mapa bibliométrico resalta un clúster (en amarillo) relacionado con la Autonomía, las Interacciones Sociales, las Aplicaciones Móviles, o los Asuntos Financieros, que destacan tanto por su volumen de publicaciones, como por su novedad cronológica. Observando su cercanía y la proyección de sus relaciones con los nodos cercanos, destacan las relaciones que surgen entre la Autonomía y la Cognición, la Evaluación, la Gestión financiera y la Competencia Financiera; si bien estas relaciones pueden parecer razonables al realizar un análisis subjetivo, el hecho de que el mapeo bibliométrico las corrobore viene a confirmar que son aspectos que deben ser tenidos en cuenta.

El análisis del mapa bibliométrico, desde el punto de vista cronológico, muestra cómo las publicaciones de artículos relacionados con la Competencia Financiera se inician en ámbitos temáticos relacionados con las Ciencias de la Salud, y que los artículos relacionados con la Educación no empiezan a tener una presencia significativa en las revistas científicas hasta poco más allá del último quinquenio. 
F. Javier Sánchez Antolín y José María Marbán Competencia financiera y educación: una aproximación desde el mapeo de la ciencia
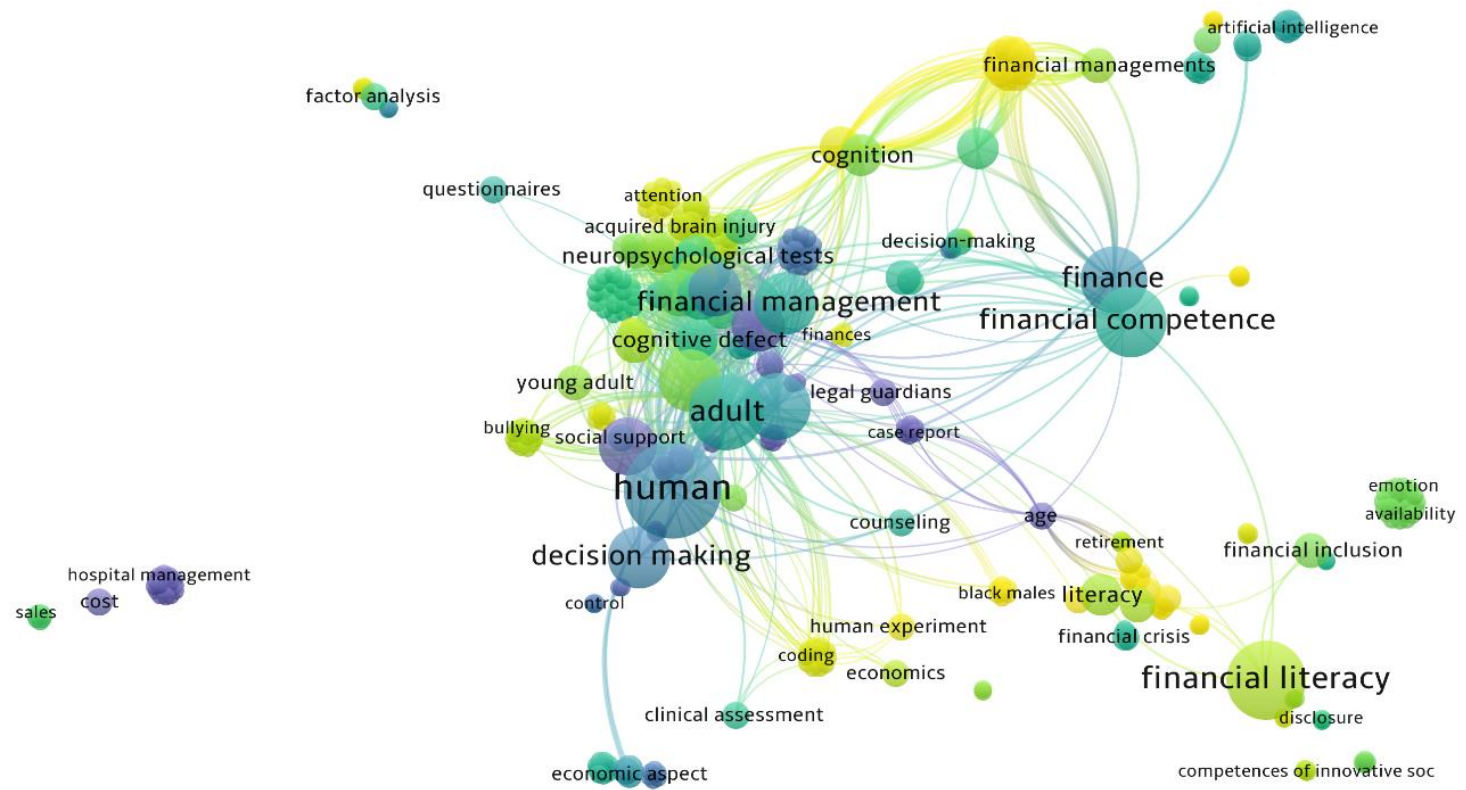

f

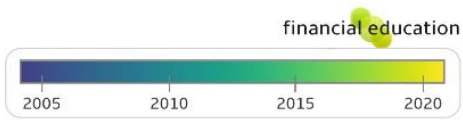

Figura 16 Competencia Financiera 1944-2021. Análisis de co-ocurrencia por conceptos clave

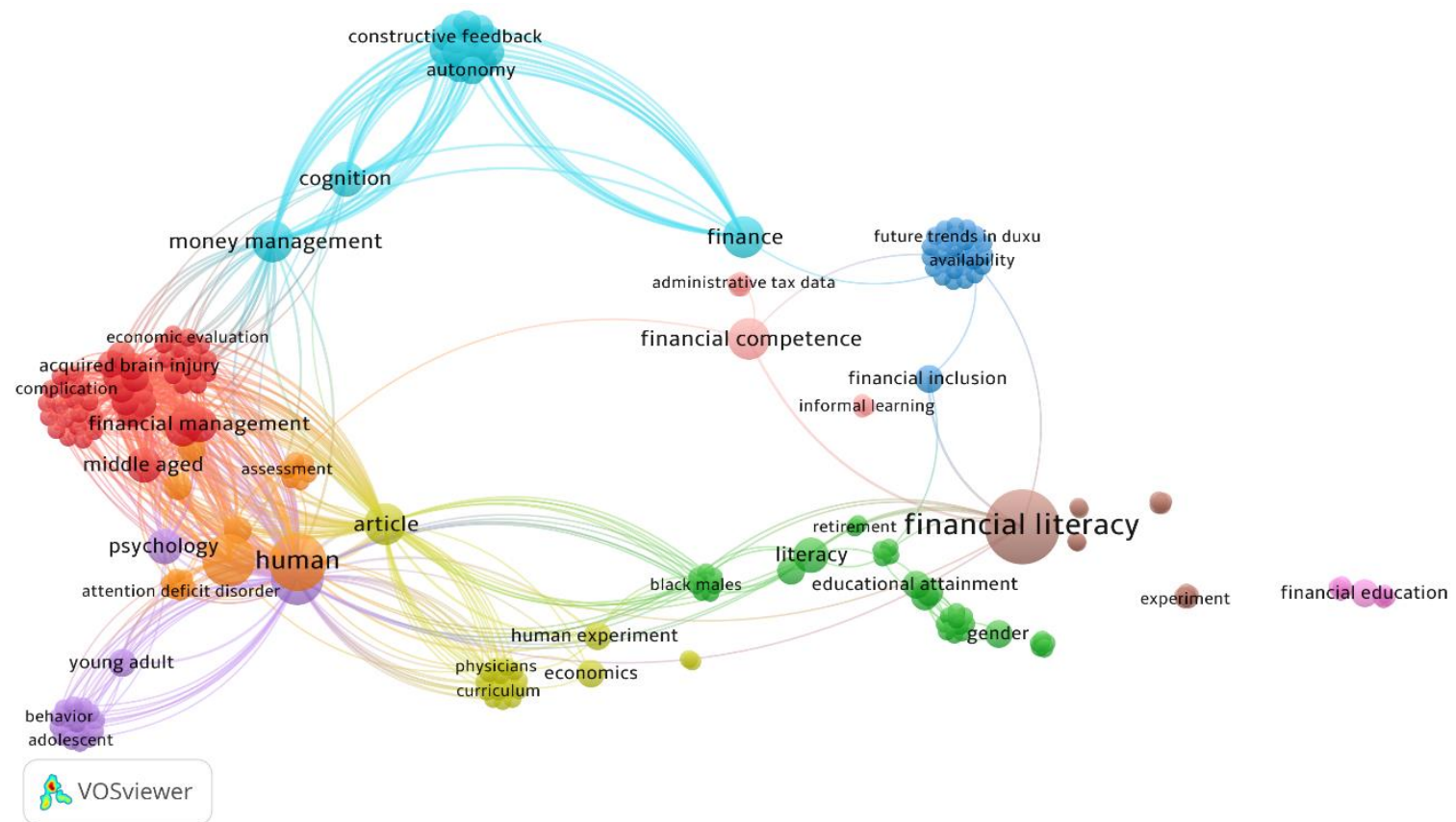

Figura 17 Competencia Financiera 2015-2021. Análisis de co-ocurrencia por conceptos clave 
Si se centra el análisis en las publicaciones realizadas entre los años 2015 y 2021 se obtiene el mapa bibliométrico de la Figura 17. Se aprecia cómo las publicaciones relativas al ámbito de la Psicología (principalmente marcadas en color verde) y la Salud (en tono rojo) siguen teniendo un volumen de publicaciones elevado sobre la Competencia Financiera, pero se identifican varios clústeres en la parte derecha relacionados con la Alfabetización Financiera y la Educación.

En el período 2016-2021 las publicaciones relacionadas con la Alfabetización Financiera suponen el 69,97\% del total y, para realizar el mapeo de las relaciones entre las palabras clave se ha optado por un filtrado, igual que en el caso anterior, representando solo aquellos conceptos con al menos diez ocurrencias (Figura 18). En una primera observación se aprecia que el volumen de nodos es muy superior al anterior, debido al mayor número de publicaciones; en segundo lugar, se advierte una estructura de clústeres similar a la anterior, con la prevalencia de tres grupos de nodos que responden, en general, a los mismos ámbitos que en el mapa de la Figura 14 correspondiente a los años 1984-2015 que son las actuaciones financieras de las personas, la Educación y los aspectos socioeconómicos.

Por lo que se refiere a la evolución de las palabras clave, destaca la separación de los nodos Alfabetización y Educación respecto al de Alfabetización Financiera, y su cercanía a los clústeres educativo y socioeconómico. También sobresale el volumen de publicaciones referidas a Finanzas y a la Educación Financiera, así como la aparición de un elevado número de nodos de su mismo clúster, lo que refleja el aumento en las publicaciones y la variedad de temáticas relacionadas con las diferentes actuaciones financieras de los individuos. 
F. Javier Sánchez Antolín y José María Marbán Competencia financiera y educación: una aproximación desde el mapeo de la ciencia

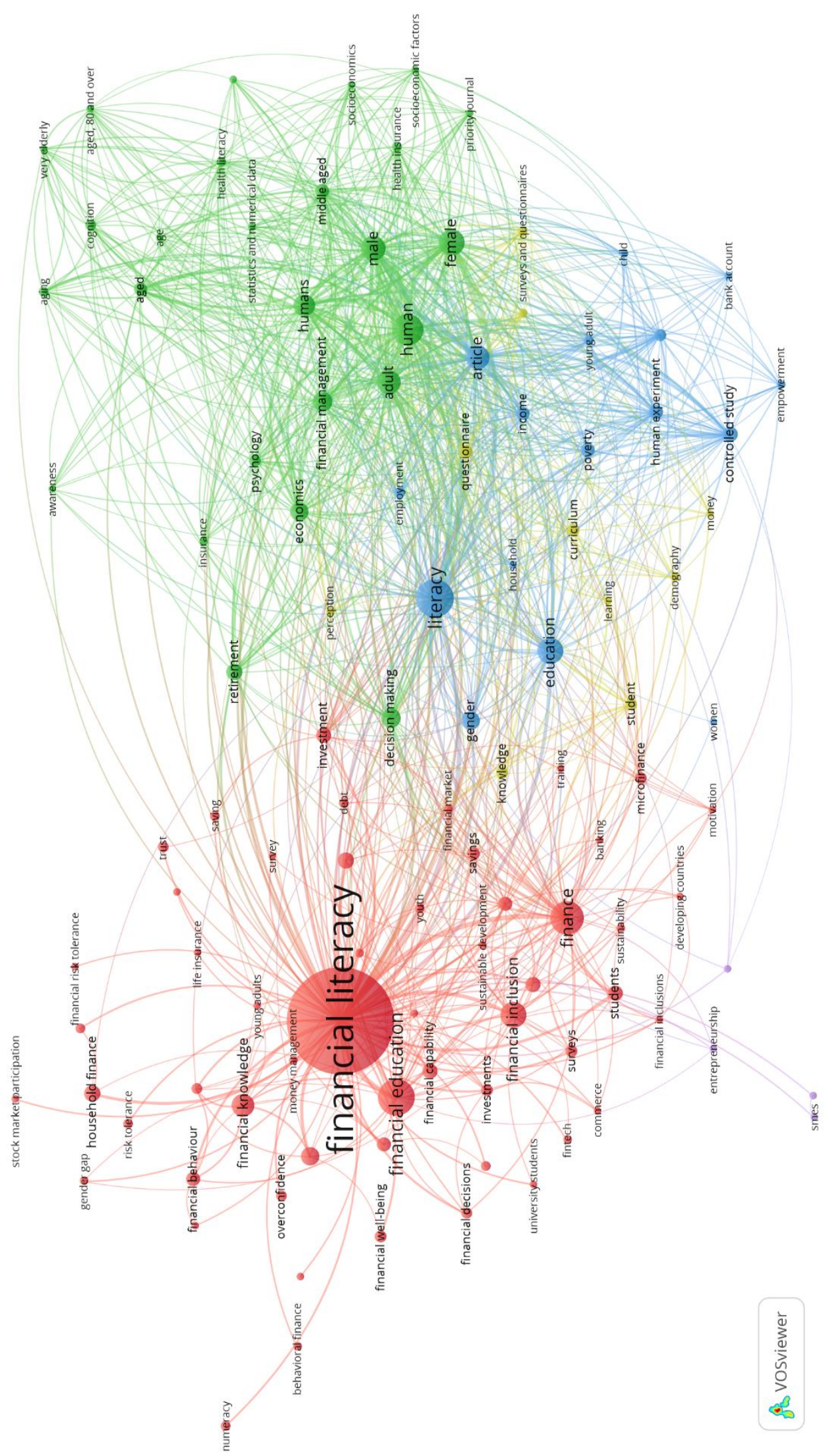

Figura 18 Alfabetización Financiera 2016-2021. Análisis de co-ocurrencia por conceptos clave 
Aislando las relaciones que generan los conceptos de Competencia Financiera y Alfabetización Financiera (Figura 19), se aprecia cómo establecen relaciones con algunos de los nodos principales de todos los clústeres, aunque llama la atención que no exista una relación importante con el tópico de Educación Financiera, tal y como lo refleja el hecho de que el algoritmo de mapeo no dibuja las relaciones entre los tres conceptos.

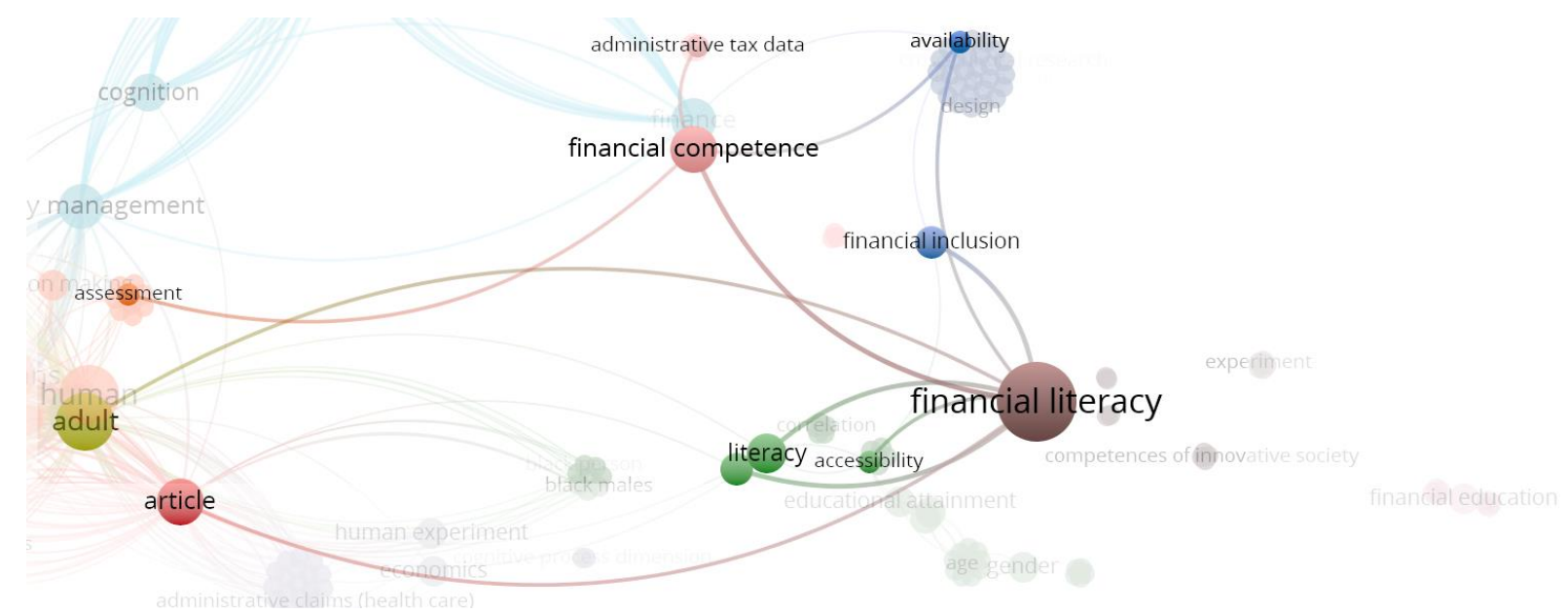

Figura 19 Competencia Financiera 2015-2021. Detalle de relaciones entre Competencia Financiera y Alfabetización Financiera

Ante la escasa relación aparente entre Competencia Financiera, Alfabetización Financiera y Educación Financiera, se realizó una nueva búsqueda por frase exacta del tópico Educación Financiera en Scopus, con un filtrado por fechas incluyendo solo las publicaciones realizadas entre los años 2015 y 2021; la búsqueda arrojó un resultado de 616 publicaciones cuyo análisis de concurrencia por conceptos clave se muestra en la Figura 20. Al aplicarle el operador booleano and con el tópico Competencia Financiera para determinar la intersección entre ambos conceptos, el resultado fue de solo 3 publicaciones. 


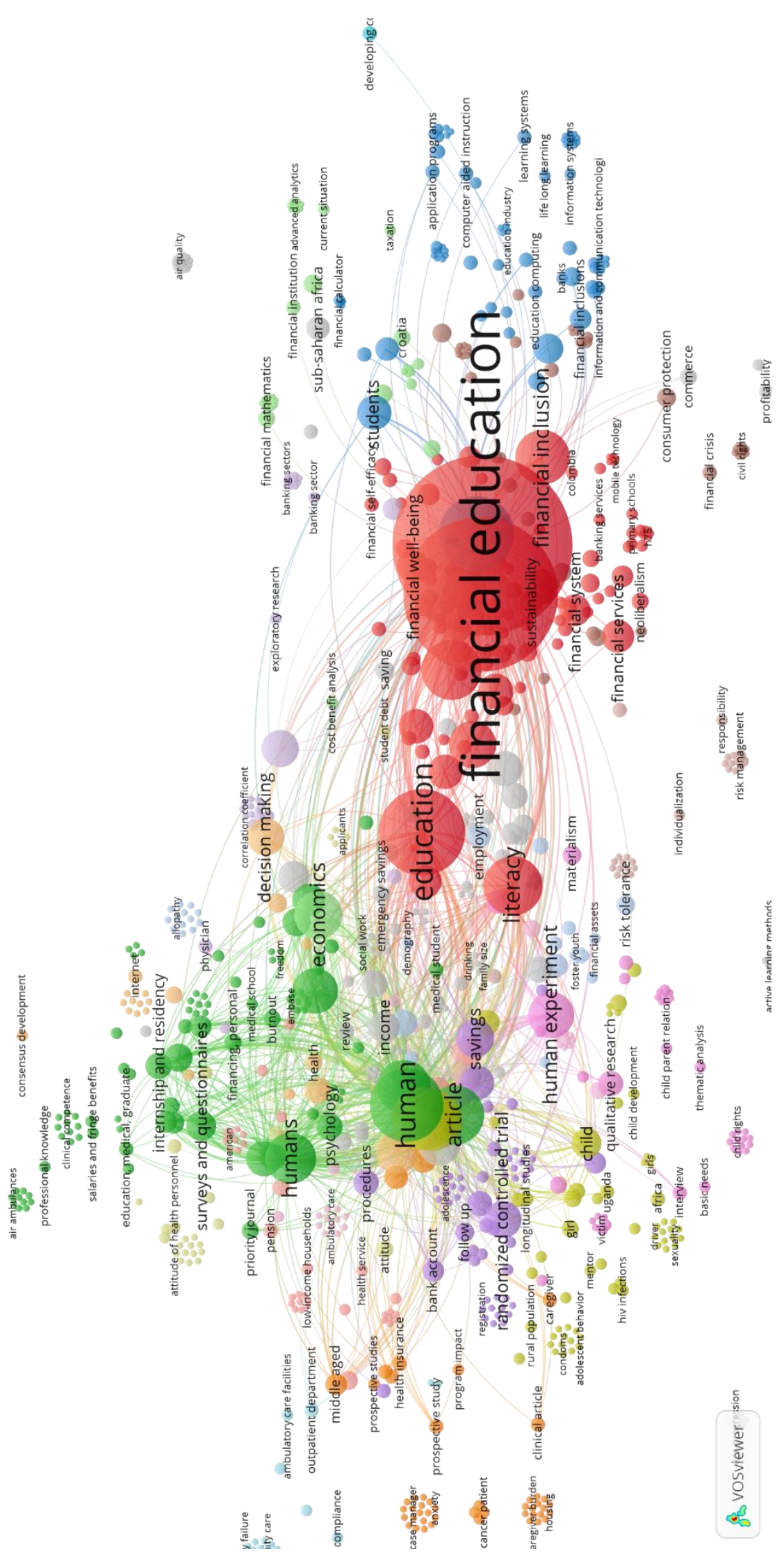

Figura 20 Educación Financiera 2015-2021. Análisis de co-ocurrencia por conceptos clave 
Aunque no es objeto de estudio de la presente revisión de la literatura, resulta sorprendente la escasa relación, en términos de palabras clave, que existe entre la Competencia Financiera y la Educación Financiera, aspecto que plantea interrogantes que merece la pena abordar en futuras investigaciones.

Al realizar un nuevo mapeo bibliométrico agrupando los artículos por país de publicación y poniéndolos en relación con las citaciones que se realizan entre dichos artículos, se puede apreciar en la Figura 21, cómo en el ámbito de la Competencia Financiera Reino Unido aparece como país con mayor número de publicaciones al inicio del período. A medida que se van publicando artículos toman relevancia también países como Australia, Italia y Estados Unidos principalmente y cómo, en los últimos años, aunque no aparecen los nombres de los países en el gráfico, los marcadores más recientes se refieren a España, Rusia y, en menor medida, Hungría.

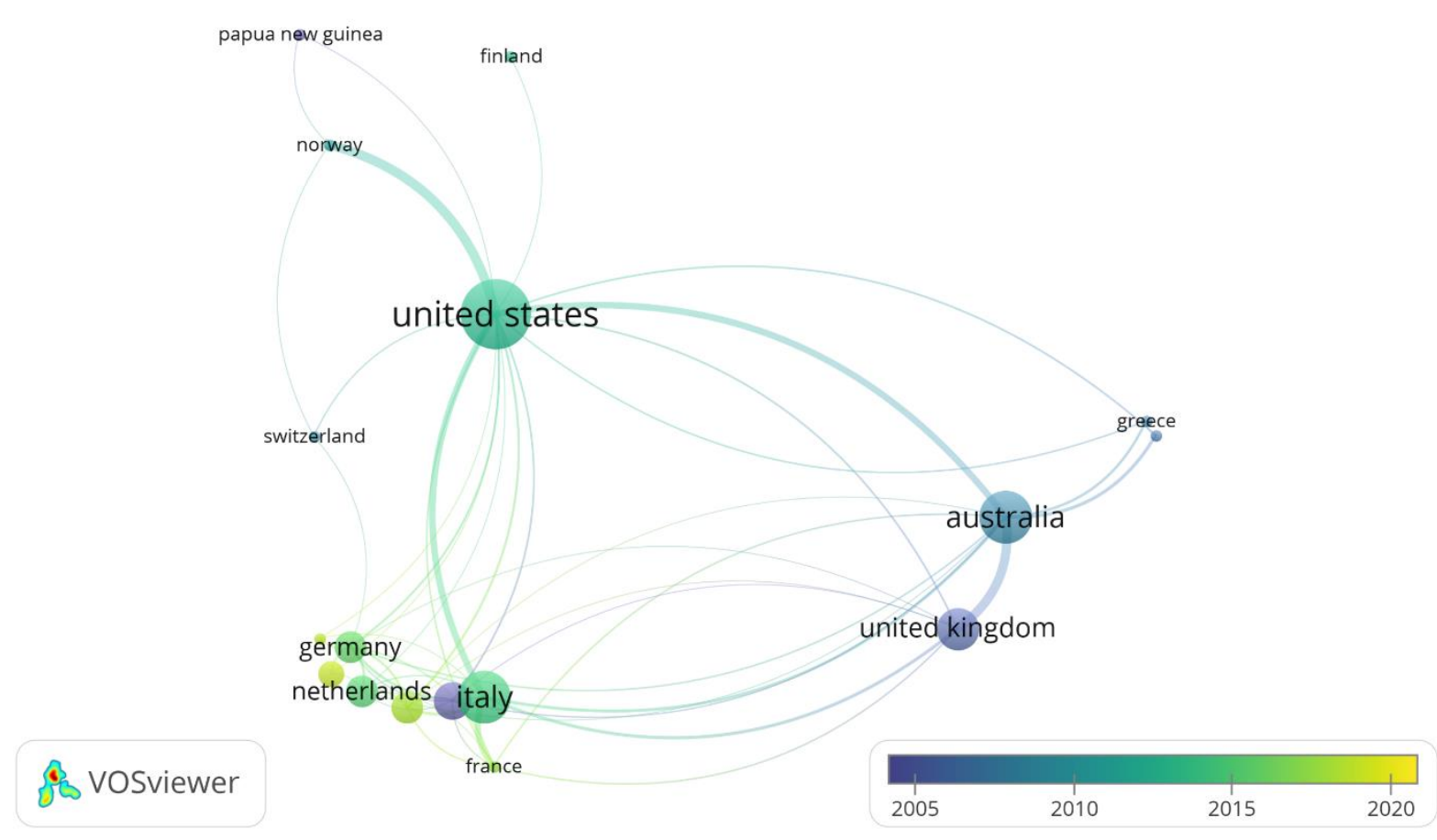

Figura 21 Competencia Financiera. Distribución de las citaciones por países y fechas de publicación

Por lo que respecta a la Alfabetización Financiera, el mapa por países refleja una amplia diversidad en los orígenes de las publicaciones, aunque predominan las publicaciones de los Estados Unidos de América. Como en anteriores mapas, aquellos países con publicaciones relacionadas se encuentran más cerca entre sí y comparten color dentro del mismo clúster, aunque no se puede encontrar un criterio de aplicación general, ya que salvo en el caso del clúster verde, en que se agrupan una serie de países próximos geográfica y culturalmente, en el resto de los casos existe demasiada variedad como para poder extraer un criterio general de relación. 


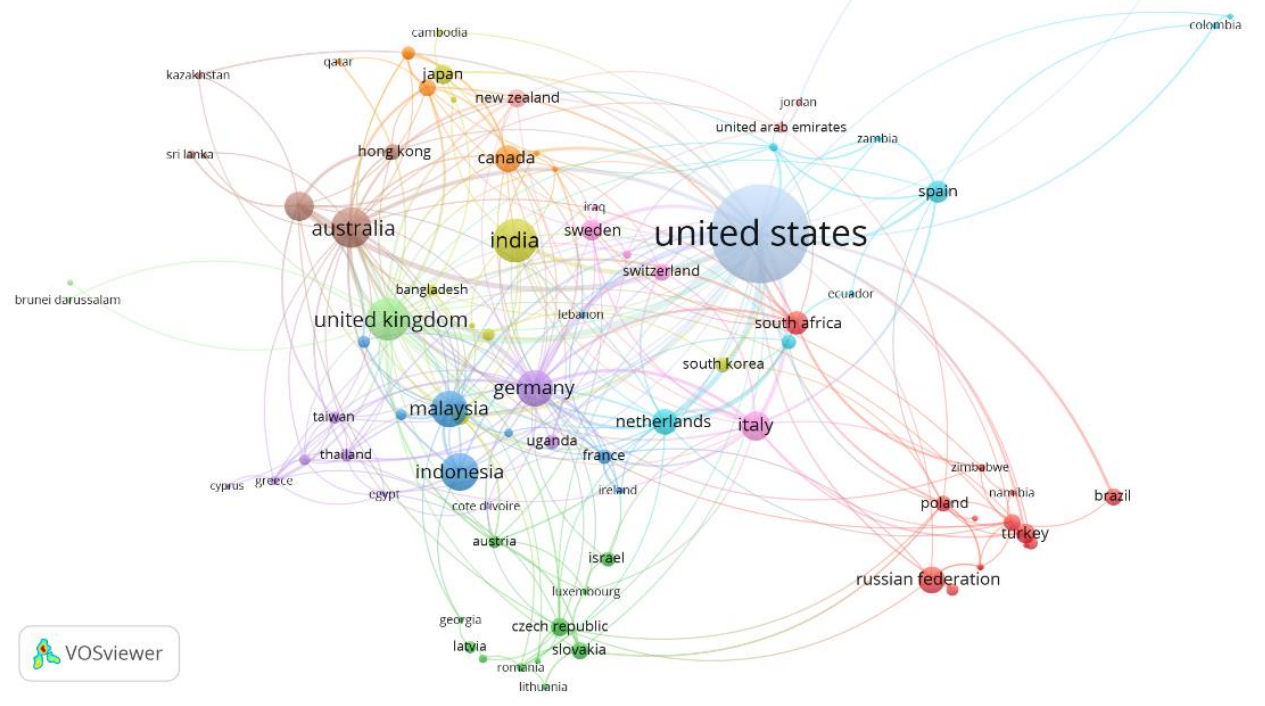

Figura 22 Alfabetización Financiera. Distribución de las citaciones por países

\section{CONCLUSIONES Y DISCUSIÓN}

Para dar respuesta a las preguntas de investigación haremos referencia a las dimensiones señaladas en el apartado de Resultados que aportan elementos de juicio para atender cada una de ellas. Así, para comprender la evolución de la investigación sobre Competencia Financiera y Alfabetización Financiera, y valorando el número de publicaciones y su evolución a lo largo de los diferentes periodos considerados, se puede concluir que hay un incremento en el interés, especialmente en el último quinquenio, en el que se han publicado casi tantos estudios (72 sobre Competencia Financiera y 2.318 sobre Alfabetización Financiera) como en los demás períodos juntos (82 y 940 respectivamente).

El interés de las preguntas de investigación por los países en los que se generan las publicaciones encuentra respuesta en el mapa bibliométrico de la Figura 21, y permite identificar que los países que comenzaron liderando la publicación de trabajos fueron los del ámbito anglosajón, especialmente los Estados Unidos de América, pero la evolución de los últimos años, reflejada en la Figura 22, está haciendo que las nuevas publicaciones tengan su origen en la Europa continental.

El análisis de las líneas de trabajo se inicia con la Tabla 2, que recoge los ámbitos científicos que publican estudios sobre la Competencia Financiera y la Alfabetización Financiera, siendo mayoritarias las Ciencias Sociales, la Medicina, y la Economía, Econometría y Finanzas en el primer caso, mientras la Economía, Econometría y Finanzas, las Ciencias Sociales y los Negocios, Gestión y Contabilidad son las disciplinas científicas que más publicaciones realizan en la segunda. 
Continuando con las líneas de investigación principales de ambos constructos, su evolución ha hecho más complejo el mapa de relaciones entre los tópicos de investigación. Mientras que en el primer período considerado (1944-2000) los temas de investigación sobre la Competencia Financiera, recogidos en la Figura 3, se agrupan alrededor de tres grandes núcleos temáticos (aspectos legales, psicológicos y clínicos), los tópicos en el último período agregado (1944-2021) de la Figura 16 muestran un mayor nivel de complejidad, produciéndose una mezcla en las relaciones entre conceptos de diferentes ámbitos de conocimiento, al tiempo que destaca el nodo de Alfabetización Financiera que, como se puede apreciar en la Figura 19, se coloca junto al de Competencia Financiera como los dos más relevantes de la literatura.

La Figura 17 y la Figura 18 centran la atención en las líneas de trabajo del último período 2015-2021, y se aprecian algunos cambios respecto a la tendencia de los períodos anteriores o a los datos acumulados:

- Aumentan en número los bloques temáticos. Surgen nuevos clústeres de conceptos relacionados con aspectos tecnológicos, económicos, sociales o educativos. En este último caso llama la atención que el clúster relacionado con la Educación Financiera, situándose cerca de la Alfabetización Financiera, apenas tenga importancia a nivel de relaciones para el algoritmo de representación de los mapas bibliométricos.

- El tópico con mayor prevalencia en este subperíodo pasa a ser la Alfabetización Financiera, que supera con claridad a la Competencia Financiera.

- Aparecen conceptos insertados en clústeres de ámbitos temáticos aparentemente alejados del suyo, como pueden ser tópicos relacionados con la Educación en clústeres de salud (por ejemplo, conceptos matemáticos o la metacognición), o los procesos de evaluación, que aparecen de varias maneras en diversos clústeres.

La última de las preguntas de investigación, que centraba su interés en la relación entre las investigaciones relativas a la Competencia Financiera, la Alfabetización Financiera y el ámbito educativo, encuentran respuesta en la Figura 20, de una forma general, y en la Figura 15 y en la Figura 19, con mayor nivel de detalle, y en todas ellas se percibe que esta relación ha ido cobrando fuerza en los últimos años, con especial preeminencia en el último período 2015-2021, años en los que las publicaciones del ámbito educativo han ido generando clústeres propios (centrados en la innovación financiera y en la valoración del riesgo y la toma de decisiones) y nuevas temáticas que se han incorporado a otros ámbitos de conocimiento (como la memoria verbal o los conceptos matemáticos en el ámbito de la salud).

Los tópicos de investigación que van ganando peso, y que podrían indicar hacia dónde se dirige la investigación en este ámbito (Figura 18), se refieren a la Educación Financiera, a las cualificaciones y titulaciones profesionales, al análisis de riesgos en diversos escenarios, a la evaluación y a la toma de decisiones.

No se puede finalizar sin hacer referencia a la sorprendente relación entre las publicaciones sobre Competencia Financiera y Educación Financiera presentada en la Figura 20; aunque los dos tópicos se encuentran próximos en el mapa bibliométrico el hecho de que no estén directamente relacionados implica que el algoritmo de VOSviewer no ha encontrado suficientes publicaciones que los relacionen; es llamativo que solo 3 publicaciones incluyan ambos tópicos entre sus palabras clave, cuando hay 616 publicaciones de Educación Financiera en los últimos años, inconsistencia que deja abierto un campo de estudio para futuros trabajos. 


\section{BIBLIOGRAFÍA}

Beltrán, G., \& Óscar, A. (2005). Revisiones sistemáticas de la literatura. Revista Colombiana de Gastroenterología, 20(1), 60-69. https://www.redalyc.org/articulo.oa?id=337729264009

Björklund, M., \& Sandahl, J. (2020). Financial literacy as citizenship education - a viable prospect? Journal of Social Science Education, 19(3), 4-20. https://doi.org/10.4119/jsse-3230

Codina, L. (2018). Revisiones bibliográficas sistematizadas: Procedimientos generales y Framework para Ciencias Humanas y Sociales. En Máster Universitario en Comunicación Social. Departamento de Comunicación. https://repositori.upf.edu/

Delors, J., \& UNESCO. (1996). La Educación encierra un tesoro / informe a la UNESCO de la Comisión Internacional sobre la Educación para el siglo XXI, presidida por Jacques Delors (Grupo Santillana de Ediciones (ed.)). UNESCO. https://unesdoc.unesco.org/ark:/48223/pf0000109590_spa

European Banking Authority. (2020). EBA Report on Financial Education 2019/2020 (EBA/Rep/2020/12).

https://www.eba.europa.eu/sites/default/documents/files/document_library/Ne ws and Press/Press Room/Press Releases/2020/EBA identifies trends and lessons learned in financial education and literacy initiatives in its second Financial Education Report/EBA F

Financial Literacy and Education Commission (U.S.). (2006). Taking ownership of the future: the national strategy for financial literacy, 2006. https://purl.fdlp.gov/GPO/LPS83756

García Peñalvo, F. J. (2017). Revisión sistemática de literatura en los Trabajos de Final de Máster y en las Tesis Doctorales. Máster Universitario en Sistemas Inteligentes, del Máster Universitario en Ingeniería en Informática y del Programa de Doctorado en Formación en la Sociedad del Conocimiento, 95. https://knowledgesociety.usal.es/sites/default/files/20170316 - Seminario SLR.pdf

Grant, M. J., \& Booth, A. (2009). A typology of reviews: An analysis of 14 review types and associated methodologies. Health Information and Libraries Journal, 26(2), 91-108. https://doi.org/10.1111/j.1471-1842.2009.00848.x

Hart, C. (1998). Doing a Literature Review: Releasing the Social Science Research Imagination. En Doing a Literature Review. SAGE Publications.

Instituto Nacional de Evaluación Educativa. (2012). PISA 2012. Competencia financiera. Informe Español.

Kosar, T., Bohra, S., \& Mernik, M. (2016). Domain-Specific Languages: A Systematic Mapping Study. Information and Software Technology, 71, 77-91. https://doi.org/10.1016/j.infsof.2015.11.001

Li, Z., Avgeriou, P., \& Liang, P. (2015). A systematic mapping study on technical debt and its management. Journal of Systems and Software, 101, 193-220. https://doi.org/10.1016/j.jss.2014.12.027

Manterola, C., Astudillo, P., Arias, E., \& Claros, N. (2013). Revisiones sistemáticas de la literatura. Qué se debe saber acerca de ellas. Cirugia Espanola, 91 (3), 149-155. https://doi.org/10.1016/j.ciresp.2011.07.009

Mundial Bank. (2010). Ministry of Finance Ministry of Finance of Russian Federation (p. 8). Mundial

Bank. 
http://documents 1.worldbank.org/curated/en/186741468304472407/pdf/FEFLOPr oject0l10StageOfinal0040810.pdf

OCDE. (2019). PISA 2021. Financial Literacy. Analytical and assessment framework. https://www.oecd.org/pisa/sitedocument/PISA-2021-Financial-LiteracyFramework.pdf

OECD. (2013). PISA 2012 Assessment and Analytical Framework: Mathematics, reading, science, problem solving and financial literacy. En OECD Report. OECD Publishing. https://doi.org/10.1787/9789264190511-en

Petersen, K., Vakkalanka, S., \& Kuzniarz, L. (2015). Guidelines for conducting systematic mapping studies in software engineering: An update. Information and Software Technology, 64, 1-18. https://doi.org/10.1016/j.infsof.2015.03.007

Ramírez-Montoya, M.-S., \& Lugo-Ocando, J. (2020). Revisión sistemática de métodos mixtos en el marco de la innovación educativa. Comunicar, 65, 9-20. https://doi.org/10.3916/C65-2020-01

Rovira, C., Codina, L., Guerrero-Solé, F., \& Lopezosa, C. (2019). Ranking by relevance and citation counts, a comparative study: Google Scholar, Microsoft Academic, WoS and Scopus. Future Internet, 11 (9). https://doi.org/10.3390/fil 1090202

Runeson, P., \& Höst, M. (2009). Guidelines for conducting and reporting case study research in software engineering. Empirical Software Engineering, 14(2), 131-164. https://doi.org/10.1007/s10664-008-9102-8

Salvo, G., Lashewicz, B. M., Doyle-Baker, P. K., \& McCormack, G. R. (2018). Neighbourhood built environment influences on physical activity among adults: A systematized review of qualitative evidence. International Journal of Environmental Research and Public Health, 15(5). https://doi.org/10.3390/ijerph15050897

Sánchez Reynoso, M. L., \& Diván, M. J. (2020). Assessment of semantic similarity in entities under monitoring: A systematic literature mapping. Revista Facultad de Ingeniería Universidad de Antioquia, Apr-Jun 20(99), 21-31. https://doi.org/10.17533/udea.redin.20200476

van Eck, N. J., \& Waltman, L. (2010). Software survey: VOSviewer, a computer program for bibliometric mapping. Scientometrics, 84(2), 523-538. https://doi.org/10.1007/s1 1192-009-0146-3

van Eck, N. J., \& Waltman, L. (2014). Visualizing Bibliometric Networks. En Measuring Scholarly Impact: Methods and Practice (pp. 285-320). Springer. https://doi.org/10.1007/978-3-319-10377-8_13

van Eck, N. J., \& Waltman, L. (2021). VOSviewer (1.6.16). Leiden University. https://www.vosviewer.com/

Westheimer, J., \& Kahne, J. (2004). What kind of citizen? The politics of educating for democracy. American Educational Research Journal, $41(2)$, 237-269. https://doi.org/10.3102/00028312041002237

Xiao, Y., \& Watson, M. (2019). Guidance on Conducting a Systematic Literature Review. Journal of Planning Education and Research, 39(1), 93-112. https://doi.org/10.1177/0739456X17723971 